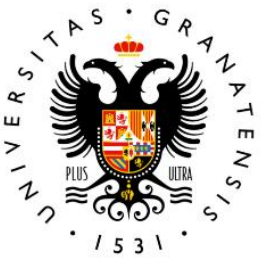

UNIVERSIDAD

\title{
DE GRANADA
}

Departamento de Didáctica de la Matemática

APLICACIONES DE LA TECNOLOGÍA EN LA RESOLUCIÓN DE UN PROBLEMA DE TRIGONOMETRÍA. UN ESTUDIO CON ESTUDIANTES DE

$1^{\circ}$ BACHILLERATO

Asunción Mora López

Granada,

Julio 2020 


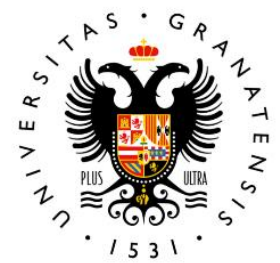

\section{UNIVERSIDAD}

DE GRANADA

Departamento Didáctica de la Matemática

\section{APLICACIONES DE LA TECNOLOGÍA EN LA RESOLUCIÓN DE UN PROBLEMA DE TRIGONOMETRÍA. UN ESTUDIO CON ESTUDIANTES DE $1^{\circ}$ BACHILLERATO}

Trabajo Fin de Máster realizado bajo la tutela del Doctor José Antonio Fernández Plaza del Departamento de Didáctica de la Matemática de la Universidad de Granada que presenta Asunción Mora López para su aprobación por el Departamento de Didáctica de la Matemática de la Universidad de Granada.

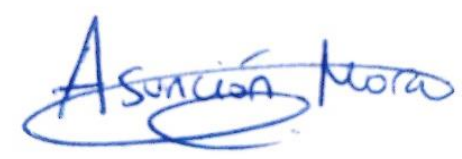

Fdo.: Asunción Mora López.

$\mathrm{V}^{\circ} \mathrm{B}^{\mathrm{o}}$ del director

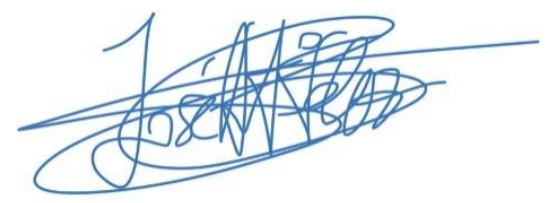

Fdo.: José Antonio Fernández Plaza. 


\section{Resumen}

Este Trabajo Fin de Máster describe un estudio exploratorio-descriptivo llevado a cabo con estudiantes de $1^{\circ}$ de Bachillerato en su modalidad científico-tecnológica con la finalidad de explorar y describir las nociones, representaciones y conceptos que los estudiantes ponen de manifiesto cuando resuelven un problema trigonométrico de forma escrita y mediante el uso de las Tecnologías de la Información y la Comunicación. El instrumento de recogida de datos es un cuestionario con preguntas de respuesta abierta y cerrada, que demanda una resolución algebraica de un problema y una reflexión sobre el empleo de un applet diseñado al efecto por los investigadores para la resolución aproximada del mismo. Para el análisis de las respuestas se ha llevado a cabo un análisis del contenido a través de una categorización de respuestas y posterior interpretación conjunta de las tareas analizadas. Con esta investigación hemos podido comprobar que el alumnado aborda correctamente la resolución algebraica de problemas trigonométricos, pero presenta dificultades en la interpretación y resolución gráfica de los mismos mediante el uso del applet diseñado al efecto.

\section{Abstract}

This Master's Thesis describes an exploratory-descriptive study carried out with 1 st Baccalaureate students in their scientific-technological modality in order to explore and describe the notions, representations and concepts that students reveal when solving a trigonometric problem in writing and through the use of Information and Communication Technologies. The data collection instrument is a questionnaire with open-ended and closed questions, which requires an algebraic resolution of a problem and a reflection on the use of an applet specifically designed by the researchers for its approximate resolution. For the analysis of the responses, an analysis of the content was carried out through a categorization the responses and a subsequent joint interpretation of the analyzed tasks. With this research, we have been able to verify that the students correctly address the algebraic resolution of trigonometric problems, but have difficulty with the interpretation and graphic resolution of these problems due to the applet designed for this purpose. 
A Salud y Juan Manuel, mis padres, por estar conmigo y apoyarme siempre

A mis hermanos por estar siempre presentes y tener confianza ciega en mis posibilidades de éxito

A mis sobrinos por la alegría que me transmiten

A todos los docentes por brindarme su orientación y conocimientos

A todas las personas que me ayudaron a culminar esta investigación de modo desinteresado 


\section{AGRADECIMIENTOS}

En primer lugar, deseo expresar mi agradecimiento al director de este trabajo, D. José Antonio Fernández Plaza, por su paciencia, dedicación y por el apoyo que me ha brindado.

Agradezco así mismo sus valoraciones, consejos y las orientaciones para la consecución de esta investigación.

Al centro de Educación Secundaria Obligatoria en el cual se ha realizado esta investigación y al profesor Nicolás Moral Moral por su apoyo y colaboración en la realización de esta investigación.

A la paciencia y amabilidad de los estudiantes que han participado en la realización del estudio, porque sin ellos no hubiese sido posible la realización del mismo.

A los docentes del máster de Didáctica de la Matemática por la valiosa formación que de ellos he recibido.

Por último, muestro mi gratitud a todos aquellos que colaboraron, de alguna forma, a la concreción de este trabajo. 


\section{ÍNDICE}

CAPÍTULO 1: PLANTEAMIENTO DEL PROBLEMA DE INVESTIGACIÓN.......... 1

1.1. Delimitación del problema y justificación de su relevancia en el área.............. 1

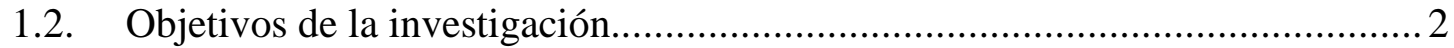

CAPÍTULO 2: FUNDAMENTACIÓN TEÓRICA …............................................... 4

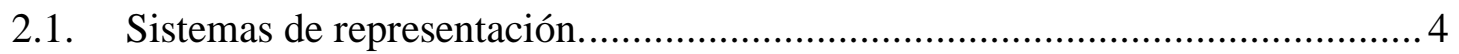

2.2. Significado de un concepto matemático escolar........................................... 8

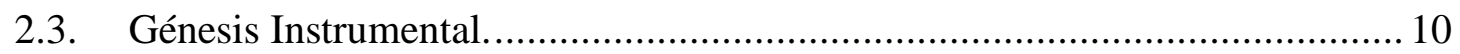

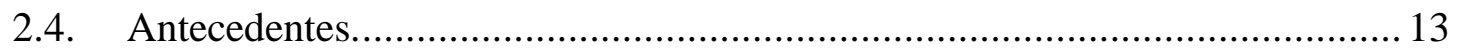

CAPÍTULO 3: METODOLOGÍA DE INVESTIGACIÓN ........................................ 18

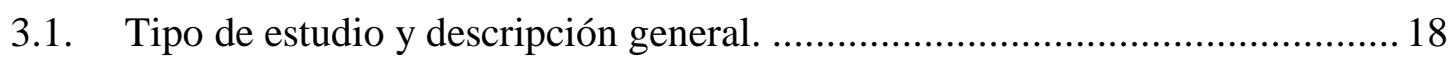

3.2. Sujetos de la investigación y variables. .................................................... 19

3.3. Elaboración del instrumento de recogida de información............................. 19

3.4. Procedimiento de aplicación del instrumento. .............................................24

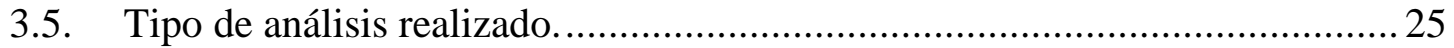

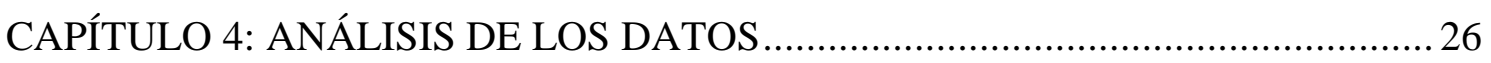

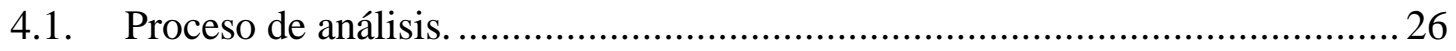

4.2. Análisis general de las respuestas del cuestionario 1..................................2 27

4.3. Análisis de casos de las respuestas del cuestionario 2 ................................ 37

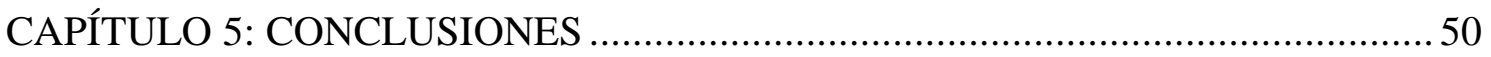

5.1. Logro del primer objetivo específico................................................50

5.2. Logro del segundo objetivo específico. .................................................. 51

5.3. Logro del tercer objetivo específico. ................................................ 52

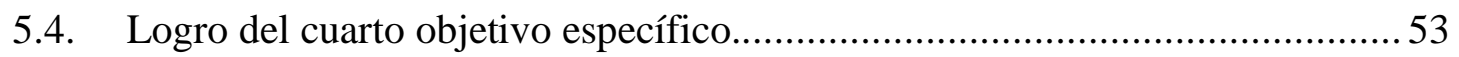

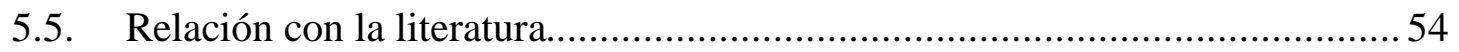

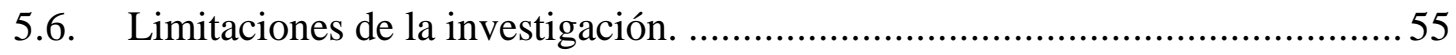

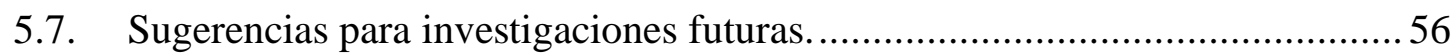




\section{ÍNDICE DE TABLAS}

Tabla 1. Respuestas de los 5 alumnos al cálculo de la distancia $\mathrm{d}_{2}$ a partir de diferentes distancias $\mathrm{d}_{1}$ dadas.

Tabla 2. Respuestas de los 5 alumnos al cálculo de la altura de la torre a partir de diferentes distancias $\mathrm{d}_{1}$ dadas.

\section{ÍNDICE DE FIGURAS}

Figura 1. Sistemas de representación de las razones trigonométricas. .............................5

Figura 2. Esquema de la situación descrita en el problema. ...................................... 6

Figura 3. Ejemplo resuelto de un problema similar al propuesto en un libro de texto de $1^{\circ}$

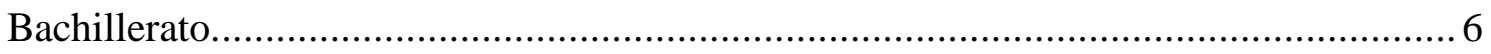

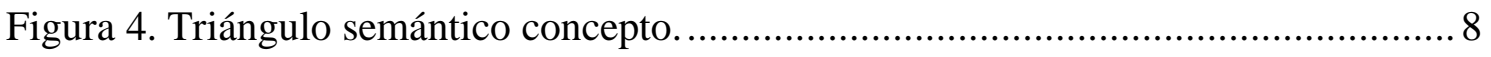

Figura 5. Triángulo semántico concepto matemático escolar. .................................... 9

Figura 6. Resolución gráfica del problema inicial propuesto mediante GeoGebra......... 13

Figura 7. Esquema de la situación descrita en el problema. ...................................... 20

Figura 8. Esquemas empleados por el alumnado en la Tarea 1...................................28

Figura 9. Ejemplo de resolución algebraica empleada por el alumnado en la Tarea 1...28

Figura 10. Fragmento de la respuesta de un alumno a la Tarea 3............................... 30

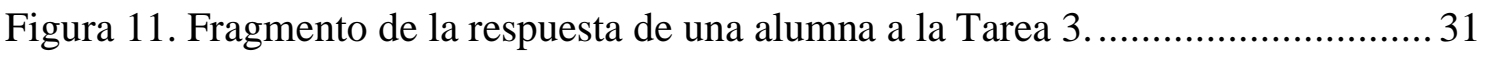

Figura 12. Respuesta de una alumna a la Tarea 4 empleando el Teorema del Coseno. . 32

Figura 13. Respuesta de una alumna a la Tarea 4 partiendo del problema resuelto....... 33

Figura 14. Respuesta de un alumno a la Tarea partiendo de las relaciones de $\mathrm{d}_{1} \mathrm{y} \mathrm{d}_{2} \ldots 33$

Figura 15. Respuesta de una alumna a la Tarea 4 que establece la relación directamente

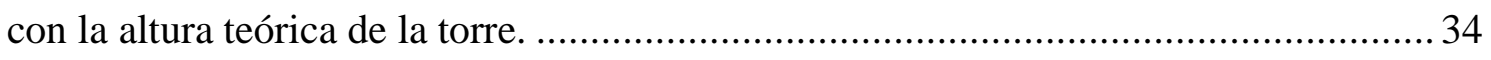

Figura 16. Respuesta de una alumna a la Tarea 4 empleando el Teorema del Seno. ..... 34 Figura 17. Respuesta de una alumna a la Tarea 4 empleando la razón trigonométrica

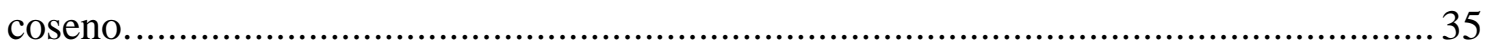

Figura 18. Respuesta de una alumna a la Tarea4 empleando la relación de $\mathrm{d}_{2}$ con la altura

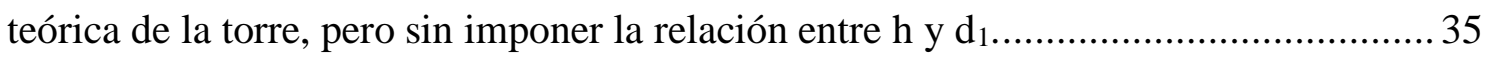

Figura 19. Captura de pantalla del problema a) realizada por una alumna.................... 37 
Figura 20. Respuesta de Carlos a la Tarea 1 expresando la relación entre la altura de la

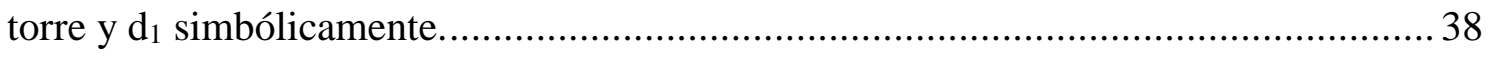
Figura 21. Respuesta de Carlos a la Tarea 2 expresando la relación entre $\mathrm{d}_{1} \mathrm{y} \mathrm{d}_{2}$ simbólicamente. 38

Figura 22. Respuesta de Carlos a la Tarea 3 identificando correctamente el radio de la circunferencia y la posición óptima de $\mathrm{O}_{2}$. 39

Figura 23. Resolución del problema a) mediante el applet realizado por Carlos. 40

Figura 24. Respuesta de Marta a la Tarea 1 expresando la relación entre la altura de la torre y $\mathrm{d}_{1}$ simbólicamente.

Figura 25. Respuesta de Marta a la Tarea 2 expresando la relación entre $\mathrm{d}_{1} \mathrm{y} \mathrm{d}_{2}$ simbólicamente.

Figura 26. Respuesta de Marta a la Tarea 3 identificando correctamente el radio de la circunferencia y la posición óptima de $\mathrm{O}_{2}$. 41

Figura 27. Respuesta de Ana a la Tarea 1 considerando que h y d covarían.

Figura 28. Respuesta de Ana a la Tarea 3. Determina algebraicamente la distancia del observador 2 a la torre en lugar de realizar la interpretación gráfica usando el applet... 43 Figura 29. Resolución del problema c) mediante el applet realizado por Ana......

Figura 30. Respuesta de Laura a la Tarea 1 expresando la relación entre la altura de la torre y $\mathrm{d}_{1}$ simbólicamente. 44

Figura 31. Respuesta de Laura a la Tarea 2 expresando la relación entre $d_{1}$ y $d_{2}$ simbólicamente. 45

Figura 32. Respuesta de Laura a la Tarea 3 identificando correctamente el radio de la circunferencia y la posición óptima del observador 2

Figura 33. Respuesta de María a la Tarea 1 expresando la relación entre la altura de la torre $\mathrm{y}_{1}$ simbólicamente. 46

Figura 34. Respuesta de María a la Tarea 2 expresando la relación entre $d_{1}$ y $d_{2}$ simbólicamente. 46

Figura 35. Respuesta de María a la Tarea 3 en la que presenta dificultades para interpretar correctamente el applet.

Figura 36. Resolución del problema a) mediante el applet realizado por María. 47 


\section{CAPÍTULO 1}

\section{PLANTEAMIENTO DEL PROBLEMA DE INVESTIGACIÓN}

En este capítulo se va a delimitar el problema de investigación del presente Trabajo Fin de Máster, justificando su relevancia en el área de Didáctica de la Matemática y se propondrán los objetivos de la investigación.

\subsection{Delimitación del problema y justificación de su relevancia en el área.}

La trigonometría del plano es un tópico muy exigente en la enseñanza de las matemáticas. Se trata de una estructura matemática de gran riqueza conceptual vinculada a otras estructuras matemáticas y enlazada con otras disciplinas. La trigonometría fusiona aritmética, álgebra, geometría y mecánica (Mathematical Association, 1950, p. 3). Además, tiene numerosas aplicaciones prácticas en ciencias y tecnología (Army, 1991; Brown, 2005).

Esto pone de manifiesto que la trigonometría se encuentra presente en multitud de fenómenos. Estos fenómenos se pueden clasificar según Mansfield (1972) en fenómenos de medida, fenómenos físicos y fenómenos relacionados con las propias matemáticas. Gracias a su elevada utilidad la trigonometría es utilizada en todo tipo de situaciones: personales, educativas, laborales, públicas y científicas (OCDE, 2017).

No obstante, es uno de los contenidos que mayor dificultad entraña para los estudiantes debido a factores diversos como son su complejidad, la conexión con numerosos fenómenos y las interconexiones con otras disciplinas (Brown, 2005). Lo anterior, unido a las distintas vías de entender, representar y relacionar sus diversas nociones básicas, como las relativas a la circunferencia goniométrica y a los triángulos rectángulos, son también algunos de los inconvenientes del aprendizaje de estos contenidos (MartínFernández, 2016).

Por otro lado, la presencia de la tecnología en las tareas cotidianas, académicas y científicas es evidente. En este sentido, la Educación Matemática no es ajena a los cambios que conlleva incorporar las nuevas tecnologías en las aulas, en efecto, existe un campo de investigación centrado en los modos de uso y en el papel que desempeñan las tecnologías en el aprendizaje y en la enseñanza de las matemáticas. 
El profesorado juega un papel relevante en la introducción de la tecnología en el aula, de hecho, el empleo de la tecnología para diseñar tareas de enseñanza y aprendizaje forman parte de su competencia profesional y de su formación inicial y continua (Domínguez Alfonso, 2011).

Existen numerosas investigaciones en relación con el estudio de la comprensión de las funciones trigonométricas y la historia de la educación de la trigonometría (MartínFernández, 2016; Bressoud, 2010; Weber, 2005). Por otro lado, también se han realizado investigaciones innovadoras con el fin de que el alumnado supere las principales dificultades que se encuentran en el proceso de aprendizaje y enseñanza de la trigonometría (Escudero, 2014; Guerrero, 2016). Sin embargo, existen muy pocas investigaciones en las que se ponga en práctica el aprendizaje y la enseñanza de la trigonometría mediante el uso de las nuevas tecnologías (Zengin, Furkan y Kutluca, 2011; Prieto, 2015; Posada-Acosta; 2017). Por ello, esta investigación pretende abrirse paso en este campo y valorar la contribución de las TIC en el proceso de enseñanza y aprendizaje de la trigonometría.

\subsection{Objetivos de la investigación.}

Este estudio propone dar respuesta a los siguientes interrogantes:

¿Cómo resuelven los estudiantes gráfica, verbal y simbólicamente un problema de trigonometría relacionado con el cálculo de la altura de una torre a partir de dos observadores? ¿Hay diferencias en el método de resolución empleado?

¿Qué conceptos y nociones trigonométricas utilizan los estudiantes para resolver el problema propuesto?

¿Cómo interpretan y responden a las cuestiones vinculadas con dichos conceptos?

¿Cómo relacionan los estudiantes la resolución manual del problema trigonométrico propuesto con la resolución mediante un applet manipulativo diseñado al efecto? ¿Cómo establecen los estudiantes las relaciones matemáticas que se generan mediante el applet? Con base en las cuestiones anteriores, establecemos el siguiente objetivo general para esta investigación: 
"Explorar y describir las nociones, representaciones y razonamientos que los estudiantes ponen de manifiesto cuando resuelven un problema trigonométrico de forma escrita y mediante el uso de un applet específico."

El objetivo general se desglosa en los siguientes objetivos específicos:

1. Diseñar e implementar un instrumento con el cual recoger información en registro verbal, simbólico y gráfico referente al problema trigonométrico propuesto.

2. Describir el método de resolución llevado a cabo por los estudiantes y establecer sus características principales.

3. Establecer relaciones entre el uso de la representación simbólica y la representación gráfica dada por el applet en la resolución del problema trigonométrico propuesto.

4. Describir el progreso realizado por los estudiantes en la resolución del problema trigonométrico planteado y en el empleo del applet relacionado. 


\section{CAPÍTULO 2}

\section{FUNDAMENTACIÓN TEÓRICA Y ANTECEDENTES}

En este capítulo desarrollaremos los referentes teóricos de esta investigación. En primer lugar, se describirán los aspectos que fundamentarán y sustentarán tanto la elaboración del cuestionario, como el análisis e interpretación de los datos y las conclusiones. Concretamente, se abordarán los siguientes aspectos: los sistemas de representación, el significado de conceptos matemáticos escolares y la génesis instrumental. Posteriormente, en el último apartado de este capítulo, profundizaremos en algunas investigaciones que constituyen los antecedentes a esta investigación.

\subsection{Sistemas de representación.}

Según Rico (2009) fue en la década de los 80 cuando se detecta un empleo sistémico de la noción de representación en Educación Matemática. En este sentido señala que "el concepto de representación se toma como equivalente a una señal externa que muestra y hace presente un concepto matemático, también como signo o marca con el que los sujetos piensan las matemáticas e, incluso, como aquellos esquemas o imágenes mentales con los que la mente trabaja sobre ideas matemáticas."

Desde entonces, Rico (2009) afirma que las representaciones se han considerado una parte esencial para analizar los procesos de aprendizaje y comprensión de las matemáticas, entendiéndolas como todas aquellas herramientas (signos o gráficos) que hacen presentes los conceptos y procedimientos matemáticos. Mediante estas herramientas los sujetos abordan e interactúan con el conocimiento matemático, es decir, pueden registrar y comunicar su conocimiento sobre las matemáticas. Radford (1998) señala que mediante el trabajo con las representaciones las personas asignan significados y comprenden las estructuras matemáticas.

Castro y Castro (1997) aporta una definición sobre representación que será la que emplearemos en este trabajo:

Las representaciones son las notaciones simbólicas o gráficas, específicas para cada noción, mediante las que se expresan los conceptos y procedimientos matemáticos, así como sus características más relevantes (...). Por representación entendemos cualquier modo de hacer presente un objeto, concepto o idea. Conceptos y procedimientos 
matemáticos se hacen presentes mediante distintos tipos de símbolos, gráficos o signos y cada uno de ellos constituye una representación.

Castro y Castro (1997) distingue, por tanto, dos familias de sistemas: las representaciones simbólicas y las representaciones gráficas. Entre las primeras se encuentran las representaciones de carácter alfanumérico. Estas se pueden simular mediante programas informáticos y su sintaxis viene descrita mediante una serie de reglas de procedimiento. Por otro lado, dentro de los sistemas de representación gráficos se encuentran las representaciones de tipo figurativo y de carácter analógico. En este caso, su sintaxis viene dada principalmente por reglas de composición y convenios de interpretación.

Por otro lado, Arenas (2012) distingue los sistemas de representación de las razones trigonométricas, estableciendo cinco tipos de representación: el verbal, el simbólico, el numérico, el gráfico y el manipulativo, tal y como se muestra en la Figura 1 siguiente.

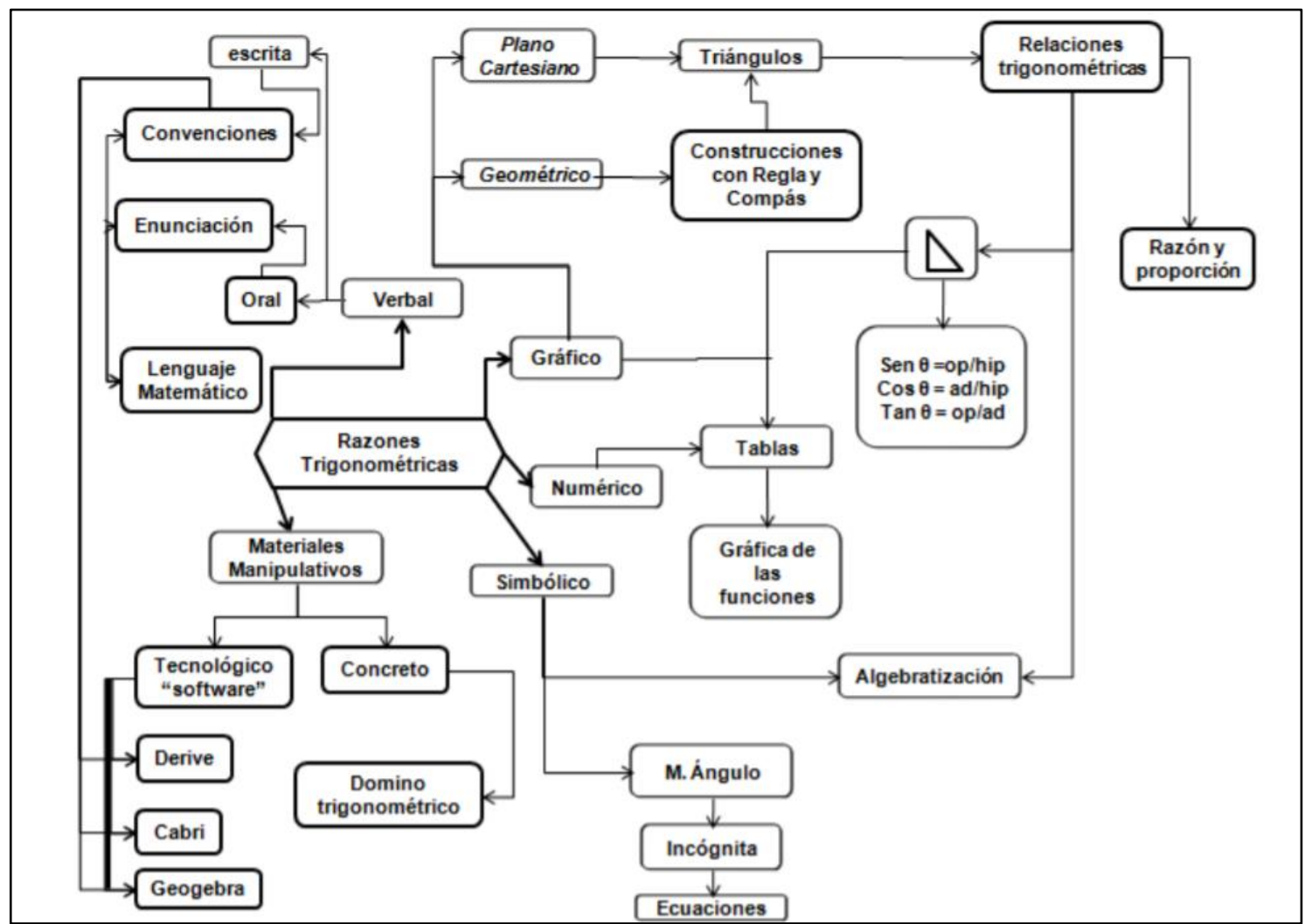

Figura 1. Sistemas de representación de las razones trigonométricas.

Para ilustrar el empleo de las representaciones simbólico-verbal y gráfica, vamos a analizar el siguiente problema, que será empleado en esta investigación. Su enunciado es el siguiente: 
"Dos observadores ven una torre desde $\alpha=70^{\circ}$ y $\beta=30^{\circ}$ respectivamente. Con el pie de la torre forman $\gamma=60^{\circ}$ y están a una distancia de $8 \mathrm{~m}(\mathrm{~d})$. ¿Cuál es la altura de la torre en metros? La siguiente ilustración muestra un esquema de la situación descrita en el problema."

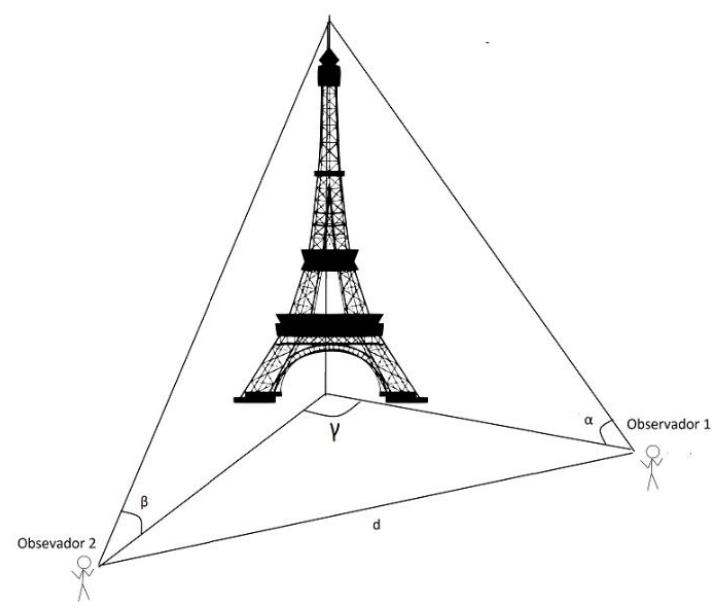

Figura 2. Esquema de la situación descrita en el problema.

En primer lugar, el esquema gráfico de la situación no destaca la condición implícita de que el pie de la torre y los dos observadores están en el mismo plano, por tanto, los triángulos que se forman son rectángulos. Esta condición es necesaria porque simplifica el problema para adaptarlo a los estudiantes de $1^{\circ}$ de Bachillerato. En segundo lugar, se trata de un problema que se presenta en los libros de texto de uso habitual (Vizmanos, 2008). En la Figura 3 se muestra un ejemplo resuelto de un problema similar al propuesto recogido en el libro de texto citado anteriormente:

Desde dos puntos $A$ y $B$ de la orilla de un rio se ve la copa de un árbol situado en la otra orilla bajo ángulos respectivos de $36^{\circ}$ y $52^{\circ}$.

La distancia que separa a $A$

de $B$ es de $3 \mathrm{~m}$, y el ángulo con

el que se ve esta distancia

desde la base del árbol es de

$95^{\circ}$.

Calcula la altura del árbol.

$$
\begin{aligned}
& \text { Los triángulos } A H C \text { y } B H C \text { son rectángulos en } \mathrm{H} \\
& \left\{\begin{array} { l } 
{ \operatorname { t g } 3 6 ^ { \circ } = \frac { h } { x } } \\
{ \operatorname { t g } 5 2 ^ { \circ } = \frac { h } { y } }
\end{array} \quad \Rightarrow \left\{\begin{array}{l}
x=\frac{h}{\operatorname{tg} 36^{\circ}} \\
y=\frac{h}{\operatorname{tg} 52^{\circ}}
\end{array}\right.\right. \\
& \text { Se aplica el teorema del coseno al triàngulo } A H B \text {. } \\
& 3^{2}=x^{2}+y^{2}-2 x y \cos 95^{\circ} \Rightarrow \\
& \Rightarrow 9=\frac{h^{2}}{\operatorname{tg}^{2} 36^{\circ}}+\frac{h^{2}}{\operatorname{tg}^{2} 52^{\circ}}-2 \frac{h}{\operatorname{tg} 36^{\circ}} \cdot \frac{h}{\operatorname{tg} 52^{\circ}} \cdot \cos 95^{\circ} \\
& \Rightarrow 9 \operatorname{tg}^{2} 36^{\circ} \operatorname{tg}^{2} 52^{\circ}=h^{2} \operatorname{tg}^{2} 52^{\circ}+h^{2} \operatorname{tg}^{2} 36^{\circ}-2 h \operatorname{tg} 36^{\circ} \operatorname{tg} 52^{\circ} \cos 95^{\circ} \Rightarrow \\
& \Rightarrow 7.78=1.64 h^{2}+0.53 h^{2}+0.16 h^{2} \Rightarrow 7,78=2.33 h^{2} \Rightarrow \\
& \Rightarrow h^{2}=3.34 \Rightarrow h \approx 1.83 \mathrm{~m}
\end{aligned}
$$

Figura 3. Ejemplo resuelto de un problema similar al propuesto en un libro de texto de $1^{\circ}$ Bachillerato. 
Por último, para su resolución algebraica se requiere emplear la razón trigonométrica tangente y el Teorema del Coseno, e interpretar adecuadamente los datos y esquema propuesto. Vamos a detallar en las siguientes líneas su resolución pautada:

- Seleccionar variables e identificar incógnitas y su notación. La altura de la torre será denotada por h, la distancia del observador 1 al pie de la torre se denotará por $\mathrm{d}_{1}$ y la distancia del observador 2 al pie de la torre se denotará por $\mathrm{d}_{2}$. El resto de variables, aún identificables en el esquema no son necesarias para su resolución. Es necesario tener en cuenta que la incógnita principal es h, y las “incógnitas auxiliares" (porque no hace falta su cálculo explícito), son $\mathrm{d}_{1} \mathrm{y} \mathrm{d}_{2}$.

- Establecer relaciones algebraicas entre las variables y reducir el proceso a la resolución de una ecuación con una incógnita con ayuda de los datos conocidos. A partir del esquema se establecen las siguientes relaciones algebraicas entre las incógnitas:

$$
\begin{gathered}
\operatorname{tg} \alpha=\frac{h}{d_{1}} ; d_{1}=\frac{h}{\operatorname{tg} \alpha} \\
\operatorname{tg} \beta=\frac{h}{d_{2}} ; d_{2}=\frac{h}{\operatorname{tg} \beta} \\
d^{2}=d_{1}^{2}+d_{2}^{2}-2 d_{1} d_{2} \cos (\gamma) ; d^{2}=\left(\frac{h}{\operatorname{tg} \alpha}\right)^{2}+\left(\frac{h}{\operatorname{tg} \beta}\right)^{2}-2 \frac{h}{\operatorname{tg} \alpha} \cdot \frac{h}{\operatorname{tg} \beta} \cdot \cos \gamma \\
d^{2}=h^{2}\left(\frac{1}{\operatorname{tg}^{2} \alpha}+\frac{1}{\operatorname{tg}^{2} \beta}-2 \frac{1}{\operatorname{tg} \alpha} \cdot \frac{1}{\operatorname{tg} \beta} \cos \gamma\right) \\
h=\sqrt{\frac{1}{\operatorname{tg}^{2} \alpha}+\frac{1}{\operatorname{tg}^{2} \beta}-2 \frac{1}{\operatorname{tg} \alpha} \cdot \frac{1}{\operatorname{tg} \beta} \cos \gamma}
\end{gathered}
$$

En resumen, se espera que los participantes en este estudio, representen gráficamente el esquema de la situación, seleccionen las variables necesarias, indaguen las relaciones algebraicas subyacentes (básicas o mediante teoremas) y expresen la ecuación con ayuda de los datos conocidos, en este caso, $\mathrm{d}, \alpha, \beta, \gamma$.

La representación manipulativa-virtual se desarrollará en el apartado de génesis instrumental, dado que implica el uso de un applet generado con GeoGebra, que el estudiante no necesariamente está familiarizado con él, por ello, lo consideramos en un 
apartado diferente, porque es objeto de este estudio indagar sobre el uso y significado matemático que dotan a este applet en relación con el problema anterior.

\subsection{Significado de un concepto matemático escolar.}

El aprendizaje del alumnado está supeditado y guiado por la construcción de significados, los cuales se realizan en su mayoría en el aula. Esta construcción de significado por parte del alumnado vendrá determinada por la forma de expresar, usar, relacionar y justificar el significado que se establece para una noción matemática.

Frege (1998, pp. 84-111) estableció la diferencia entre signo y significado, incluyendo dentro de este último una distinción entre sentido y referencia. Llevando a cabo una actualización de los primeros triángulos semánticos, la noción de significado vendría esquematizada según la Figura 4 que se muestra a continuación.

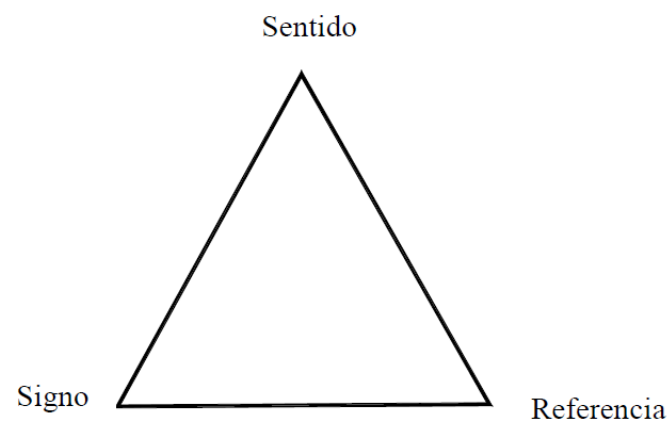

Figura 4. Triángulo semántico concepto.

En el triángulo mostrado, la referencia se refiere a los aspectos formales que evalúan la veracidad de las proposiciones derivadas del concepto propiamente dicho, el signo hace referencia al término o señal mediante el que se expresa, y su sentido hace referencia a la forma de darse de ese objeto.

Por otro lado, Rico (2012) determina el significado de un concepto matemático escolar considerando tres componentes. Estas componentes son las siguientes:

- Los sistemas de representación: hace referencia a los signos, los gráficos y las reglas que hacen presente al concepto y lo relacionan con otros.

- La estructura conceptual: incluye los conceptos y las propiedades, los argumentos y las proposiciones y los criterios de veracidad que de ellos se derivan.

- La fenomenología: incluye todos los fenómenos, los contextos y las situaciones en los que se presenta el concepto o pueden darle sentido. 
Esta noción de significado vendría esquematizada según la Figura 5 que se muestra a continuación.

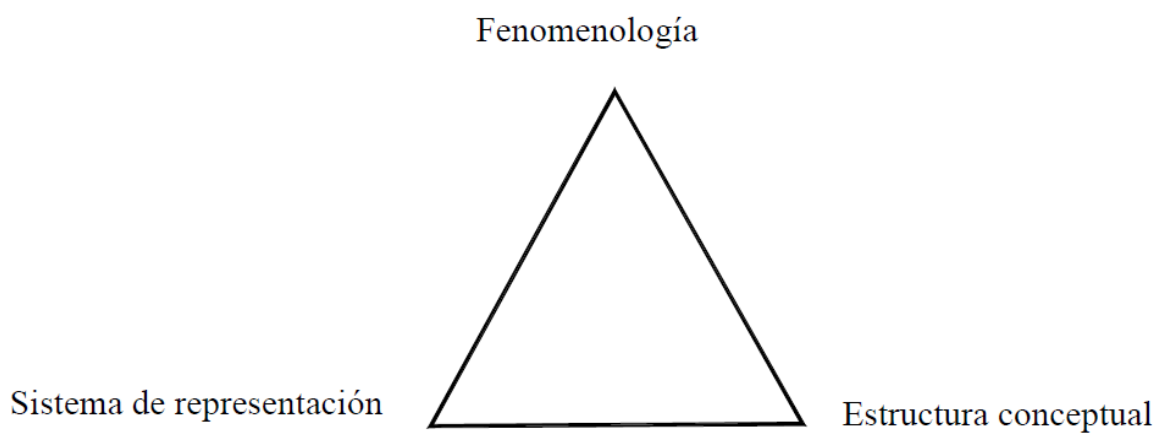

Figura 5. Triángulo semántico concepto matemático escolar.

Esta noción de significado será la que usaremos en esta investigación para abordar el significado de la razón trigonométrica tangente.

A continuación, vamos a abordar el significado de la razón trigonométrica tangente a partir de sus tres componentes: estructura conceptual, sistemas de representación y sentido/modos de uso.

- En la estructura conceptual de la razón trigonométrica tangente encontramos su definición mediante un triángulo rectángulo arbitrario, su extensión a la circunferencia goniométrica que permite definirla para ángulos mayores de $90^{\circ}(\pi / 2$ radianes) y su función definida $\mathbb{R}-\left\{\frac{\pi}{2}+\kappa \pi\right\}$, con $\mathrm{k}$ entero, que es periódica de periodo $\pi$. Sus propiedades son las siguientes: $\lim _{x \rightarrow \frac{\pi}{2}} \tan (x)= \pm \infty ; \tan (\alpha)=-\tan (\pi-\alpha) ; \tan (\alpha)=1 / \tan \left(\frac{\pi}{2}\right.$ $\alpha) ; \tan (\alpha)=-\tan (-\alpha)$

- En los sistemas de representación encontramos la representación verbal mediante la palabra "Tangente", la representación simbólica mediante "tan" o "tg", y su definición $\tan (x)=\frac{\operatorname{sen}(x)}{\cos (x)}$ y la representación gráfica en el triángulo rectángulo, en la circunferencia goniométrica con los signos correspondientes y en la función real definida en $\mathbb{R}-\left\{\frac{\pi}{2}+\kappa \pi\right\}$ con $\mathrm{k}$ entero.

- En los modos de uso la tangente tiene significado de pendiente de una recta, como la tan $(\alpha)$ que es el ángulo que forma la recta con el eje X. Además, la tangente resulta útil para resolver problemas en diferentes ámbitos tales como astronomía para el cálculo del radio de la Tierra, distancia de la Tierra a la Luna y distancia de la Tierra al Sol; cartografía 
para la elaboración del mapa de un lugar del que se conocen algunas distancias y algunos ángulos; construcciones para el diseño de un edificio que cumpla ciertas exigencias de orientación y la navegación para la construcción de cartas marinas (Mateo, Sánchez, y Valera, 1996; Plazas Rojas, 2012; Rodríguez Arós, Blanco, y Muiños, 2012).

\subsection{Génesis Instrumental.}

La disponibilidad de un artefacto que tiene la capacidad de satisfacer las necesidades de un sujeto no es suficiente para que sea usado con el fin adecuado. Por ello, el profesorado tiene un papel muy importante en este sentido para que los estudiantes usen los artefactos en sus procesos de aprendizaje de las matemáticas y aprendan a resolver problemas a través de ellos. La génesis instrumental hace referencia a la construcción progresiva de uso de un artefacto por un sujeto, con un propósito concreto y en un ambiente específico (Trouche, 2004).

El Enfoque Instrumental aborda la dimensión tecnológica de la Educación Matemática, articulando los aspectos importantes de la integración tecnológica en el proceso de enseñanza y aprendizaje de las matemáticas. Como base teórica para analizar el proceso de Génesis Instrumental de los estudiantes utilizaremos el Enfoque Instrumental de Rabardel (1995).

Este enfoque estudia la transformación de artefacto a instrumento y los procesos que envuelven esa transformación progresiva. En toda situación de actividad o de utilización de artefactos, instrumentos, existe siempre tres elementos relacionados: el sujeto, el instrumento y el objeto, siendo el instrumento un intermediario entre el sujeto y el objeto.

De esta manera, al autor señala que:

- Sujeto: hace referencia a un individuo o grupo de individuos que desarrollan una acción y/o son elegidos para el estudio y el desarrollo de sus posibles esquemas de utilización.

- Artefacto: se entiende como algo susceptible de su uso, elaborada para inscribirse en actividades intencionales como, por ejemplo, un medio material como un ordenador o un medio simbólico como el lenguaje algebraico o un gráfico en un sistema de coordenadas entre otros.

- Instrumento: se entiende como un artefacto en situación de uso. Por ello, la noción de instrumento involucra tanto al artefacto como a los esquemas mentales 
(esquemas de utilización) desarrollados por el sujeto cuando realiza una serie de tareas.

Por otro lado, Rabardel (1995) define los esquemas de utilización como el conjunto estructurado de las características generalizables de la acción que permiten repetir la misma acción o aplicarlas en nuevos contextos. Estos esquemas se clasifican a su vez de la siguiente manera:

- Esquemas de uso: relativos a unas operaciones, es decir, a la manipulación técnica del artefacto.

- Esquemas de acción instrumental: relativos a la utilización de un artefacto en vista de realizar una acción.

Además, el autor señala que la utilización de un artefacto no es neutral para el sujeto ya que éste realiza una actividad cognitiva de construcción o de evolución de esquemas de utilización a medida que hace uso del artefacto.

Por ello, dado que el instrumento se compone del artefacto y de los esquemas de utilización relacionados con éste, se concluye que el instrumento es específico a un sujeto ya que los esquemas correspondientes dependen de sus conocimientos. Así, se produce una evolución en el sujeto durante el curso de las tareas, volviendo a invertir esquemas de utilización familiares ya constituidos o produciendo nuevos esquemas de uso que le permiten alcanzar los objetivos contemplados. Este proceso recibe el nombre de Génesis Instrumental.

Durante este proceso, el sujeto se apropia de los instrumentos, confiriéndoles funciones que van más allá de sus funciones constitutivas. De esta manera, el sujeto puede elaborar sus instrumentos utilizando las potencialidades del artefacto. Así, la Génesis Instrumental depende del artefacto y también de la utilización que hace el sujeto. Este proceso consta de dos dimensiones:

- Instrumentalización: es la parte artefactual del instrumento y hace referencia al enriquecimiento de las propiedades del artefacto por parte del sujeto. Es decir, es el resultado de la atribución de una función al artefacto por parte del sujeto.

- Instrumentación: hace referencia a la asimilación de nuevos artefactos a los esquemas y la acomodación de los esquemas para dar nuevos significados a los 
artefactos. Se produce la construcción de esquemas de uso por parte del sujeto, relativos a la ejecución de ciertas tareas.

Rabardel (1995) señala que la combinación de estos dos procesos conduce a la reorganización de los esquemas de utilización produciendo como consecuencia una modificación del instrumento.

Vamos ahora a describir teóricamente el applet diseñado por el equipo de investigación para apoyar la resolución algebraica del problema. La potencialidad de este applet reside en que sin emplear teoremas avanzados de trigonometría (Teorema del Coseno/Seno), el estudiante puede aproximar la solución real del problema anteriormente descrito.

El enlace al mismo es el siguiente: https://www.geogebra.org/m/bwretzcp.

Esta aplicación modela la situación de manera dinámica proyectando la realidad tridimensional en el plano. La torre y su pie quedan ubicados en el origen de coordenadas en el punto A. Se ubica en el eje X al observador 1 como un punto libre, es decir, podemos variar arbitrariamente su distancia $\left(\mathrm{d}_{1}\right)$ al pie de la torre (punto $\left.\mathrm{A}\right)$. Con ayuda del dato que proporciona el ángulo que forman los dos observadores con el pie de la torre $(\gamma)$, se puede representar la recta en la que tendrá que estar ubicado el observador 2 a una conveniente distancia $\mathrm{d}_{2}$, cuya posición no es libre, sino que dependerá del parámetro $\mathrm{d}_{1}$ y los datos conocidos de los ángulos de visión respectivos $\alpha$ y $\beta$. A continuación, el applet responde a la siguiente pregunta básica: ¿Cuál sería la altura de la torre si se observara desde una determinada distancia y con cierto ángulo?, al expresar directamente $h=d_{1} \cdot \operatorname{tg} \alpha$, donde $\mathrm{d}_{1}$ varía libremente. A partir de esta condición expresamos también $d_{2}=\frac{h}{\operatorname{tg} \beta}=\frac{d_{1} \cdot \operatorname{tg} \alpha}{\operatorname{tg} \beta}$. Por tanto, el observador 2 queda unívocamente expresado en función de $\mathrm{d}_{1} \mathrm{y}$ los ángulos fijos $\alpha$ y $\beta$. Hay una condición que aún no se ha impuesto, que es la distancia entre los dos observadores d. Se marca esa distancia mediante una circunferencia de centro el observador 1 y radio d. Entonces la solución aproximada a la altura real será aquella disposición visual en la que el observador 2, manipulando convenientemente el observador 1, se situará en la circunferencia prefijada. No obstante, el applet previamente usando internamente el Teorema del Coseno (que no hace falta que el estudiante tenga conocimiento previo de él), calcula la altura teórica de la torre que la facilitará al estudiante con el fin de que este valore cuán cerca está la aproximación de su valor real. 
A modo de ejemplo, en la Figura 6 que aparece a continuación mostramos la captura de pantalla del problema anterior propuesto resuelto con la aplicación:

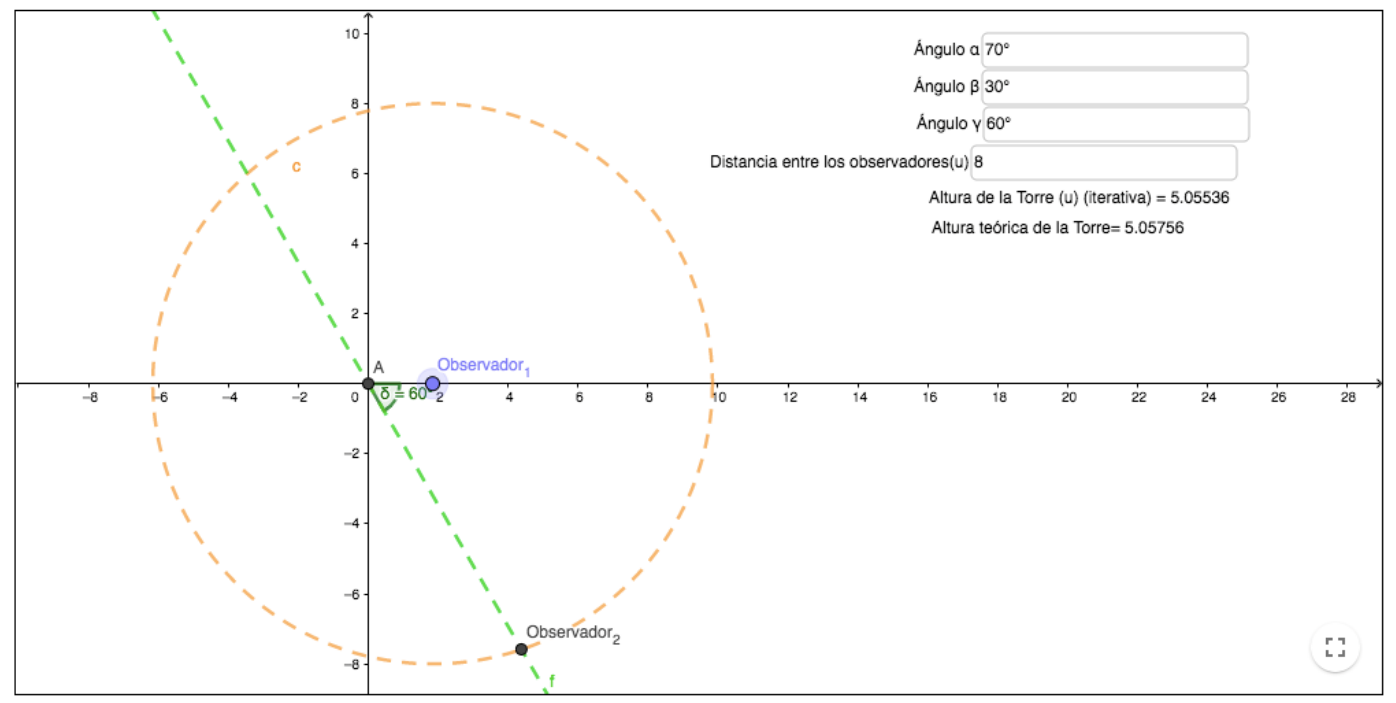

Figura 6. Resolución gráfica del problema inicial propuesto mediante GeoGebra.

Se puede observar que la altura aproximada calculada por la aplicación es de 5,055 m, muy cercana a la altura teórica que es de 5,058 m.

En resumen, el applet permite a priori realizar una aproximación al problema algebraico formal, dado que el Teorema del Coseno no es necesario para estimar la solución aproximada al mismo. La posterior resolución algebraica permitirá comprobar el ajuste de la solución aproximada dada por el applet.

Sin embargo, el applet presenta limitaciones de cara a su uso didáctico. La primera de ellas, es que no es intuitivo para el alumnado, de hecho, reflejaremos evidencias de que los estudiantes no comprenden espontáneamente su uso, por lo que se requerirá una cierta pauta. En segundo lugar, presenta una característica técnica de que la precisión de la aproximación dependerá en gran medida de aplicar convenientemente el zoom para minimizar los errores en cuanto el ajuste del observador 2 a la circunferencia "óptima" y, por tanto, en el ajuste de la altura iterativa con la altura real de la torre.

\subsection{Antecedentes.}

Weber (2005) señala que, aunque la trigonometría es uno de los primeros tópicos que vincula al álgebra, a la geometría y al razonamiento gráfico, no se le ha prestado la atención que merece. Markel (1982) también manifestó su preocupación por la escasa atención que se le dedicaba a la trigonometría en la educación secundaria, de modo que 
el alumnado presentaba grandes lagunas en los conceptos trigonométricos fundamentales a su llegada a la universidad.

A continuación, se va a presentar y describir algunas de las principales investigaciones centradas en el aprendizaje de la trigonometría clasificadas en las categorías siguientes:

- Investigaciones sobre las técnicas para la enseñanza de la trigonometría.

- Investigaciones sobre el uso de las nuevas tecnologías para el aprendizaje de la trigonometría.

- Investigaciones sobre el estudio de las dificultades y errores en el aprendizaje de la trigonometría.

- Investigaciones sobre los significados de las razones trigonométricas que manifiestan los estudiantes.

Pasamos a detallar los estudios ubicados en cada una de estas líneas de investigación.

\section{Investigaciones sobre las técnicas para la enseñanza de la trigonometría.}

Kendal y Stacey (1998) investigan las diferencias entre dos aproximaciones para la introducción de la trigonometría al alumnado: la basada en la relación entre longitudes de los lados en un triángulo rectángulo y la aproximación mediante la circunferencia unidad. En vista de los resultados, sugieren que la trigonometría sea introducida mediante el uso de la circunferencia unidad, conectando ésta a la definición como cociente, que es la utilizada para la resolución de triángulos rectángulos.

Thompson (2007) investiga cómo el emplear situaciones reales como punto de partida para planificar una unidad didáctica sobre trigonometría podría favorecer la comprensión de los estudiantes. También, intenta hallar una aproximación alternativa a dicho contenido.

Bressoud (2010) utiliza la historia de la trigonometría para razonar cómo debería ser enseñada, si mediante un triángulo rectángulo o bien mediante la circunferencia goniométrica. Finalmente, concluye que la introducción de la trigonometría mediante el triángulo rectángulo es un atajo en la enseñanza y aprendizaje de la misma y tiene sus contraindicaciones. 


\section{Investigaciones sobre el uso de las nuevas tecnologías para el aprendizaje de la trigonometría.}

Army (1991) investiga una aproximación a la enseñanza de la trigonometría usando situaciones reales y calculadora gráfica en la enseñanza universitaria. Demostró que el uso de la calculadora gráfica tenía un efecto positivo en la enseñanza de la trigonometría.

Blackett y Tall (1991) examinan los beneficios del uso de software interactivo con gráficos en trigonometría, el cual debiera facilitar a los estudiantes establecer relaciones numéricas y geométricas. El experimento confirmó que este tratamiento ayudaba al grupo experimental a mejorar sus resultados al compararlos con un grupo de control.

Zengin, Furkan y Kutluca (2011) determinan los efectos del software GeoGebra en la comprensión de la trigonometría por parte de los estudiantes. Se empleó para ello dos grupos, uno experimental, que se sometió a sesiones con dicho software, y otro de control. Se demostró que existe una clara diferencia entre los grupos. El grupo experimental que asistió a las sesiones con el software obtenía mejores resultados que el grupo de control. El estudio muestra que una instrucción asistida con dicho software, como suplemento para promover un aprendizaje constructivista, es más efectivo que una simple metodología constructivista.

Prieto (2015) describe una secuencia de análisis geométrico del comportamiento de los signos de las razones seno, coseno y tangente de un ángulo por medio del software GeoGebra debido a que en los últimos años el estudio de la trigonometría se ha convertido en un proceso memorístico y mecánico. La finalidad del autor es dotar de sentido a los signos de las razones trigonométricas en los distintos cuadrantes del plano cartesiano, considerando la noción de razón trigonométrica de un ángulo desde una perspectiva geométrica y vinculada a una circunferencia unitaria.

Posada-Acosta (2017) investiga las potencialidades del software GeoGebra en la enseñanza de la trigonometría. Se demostró que el alumnado fue capaz de inferir regularidades a partir de un triángulo rectángulo con propiedades dinámicas. Esto repercutió favorablemente en la motivación del mismo para la aplicación de la informática en el proceso docente, así como para el estudio de la geometría y la trigonometría. 


\section{Investigaciones sobre el estudio de las dificultades y errores en el aprendizaje de la trigonometría.}

Existen investigaciones que ponen de manifiesto la casi inexistencia de la resolución de problemas en la trigonometría, centrando las actividades principales en la presentación de objetos geométricos y sus propiedades. Las representaciones estereotipadas sobre conceptos trigonométricos que se realiza en la mayoría de las aulas no hacen más que generar en el alumnado ciertas dificultades para comprender la generalidad y la particularidad de la trigonometría.

Héctor Ponce (2000) afirma que mientras que, para otros conocimientos, la enseñanza y el aprendizaje de las matemáticas se basan en la resolución de problemas, en el trabajo con trigonometría parecen inexistentes, centrando las actividades en la presentación de los objetos geométricos y sus propiedades.

Berthelot y Salin (1994) afirman que "las características de la enseñanza de la trigonometría es la presentación ostensiva de los objetos geométricos”. Así, es el docente el que presenta directamente los conocimientos geométricos apoyándose en la observación de una realidad sensible o de una representación, suponiendo que el alumnado es capaz de apropiarse del contenido y de entender su aplicación en otras situaciones.

Itzcovich (2005) considera que la trigonometría es un aliado para que el alumnado se vincule con una manera específica de producir y validar relaciones. Aunque algunas propiedades se aceptan como punto de partida, esto no significa que se enuncien sin ninguna interacción con ellas, sino que se realiza con la finalidad de que el alumnado interactúe con los problemas para poder enunciarlas después. Por ello, las situaciones que se proponga al alumnado con la finalidad de indagar, identificar o reconocer propiedades de la trigonometría deben impactar en procesos intelectuales que permitan hacer explícitas las características y las propiedades de los objetos geométricos, más allá de las representaciones gráficas de dichas situaciones.

A continuación, mostramos algunas investigaciones que se han centrado en la búsqueda de las principales de dificultades y errores en relación con la trigonometría.

Escudero (2014) propone identificar los errores matemáticos, incluidos errores relacionados con trigonometría, cometidos por el alumnado de Bachillerato de un centro 
de secundaria durante un curso escolar para poder conocer su tipología. Como resultado se obtienen diferentes categorías, que pueden ser utilizadas como herramienta que ayude a identificar los errores y a seleccionar estrategias adecuadas para superar dichas dificultades.

Guerrero (2016) realiza una investigación con el fin de identificar y clasificar los errores y dificultades al abordar situaciones problema de resolución de triángulos que involucren el uso de el Teorema del Seno y el Coseno. Se concluye que los errores presentados en problemas con resolución de triángulos, tienen una estrecha relación con la aritmética, la geometría y el álgebra.

Dina Kamber y Djurdjica Takaci (2018) investigan sobre la comprensión de los estudiantes de los conceptos trigonométricos en el marco de los contextos de trigonometría de triángulos y círculos, así como la transición entre estos dos contextos. Se concluye que los estudiantes tienen dificultades con las propiedades de periodicidad y el hecho de que las funciones trigonométricas no sean individuales. Además, existe una comprensión deficiente de la medida en radianes y una falta de conexión con el círculo unitario.

\section{Investigaciones sobre los significados de las razones trigonométricas que manifiestan los estudiantes.}

Martín-Fernández (2016) realizó un estudio exploratorio para determinar los significados puestos de manifiestos por los estudiantes respecto al concepto de razón trigonométrica seno y coseno. El objetivo era indagar sobre las representaciones, conceptos, nociones y sentidos que los estudiantes emplean cuando se les pide describir o mostrar el seno y el coseno de un ángulo. Los resultados muestran varios tipos de representaciones y sentidos, algunos ya reconocidos en otros estudios, junto con otros nuevos.

Martín-Fernández (2019) realizó un estudio exploratorio para determinar los significados puestos de manifiestos por los estudiantes respecto al concepto de razón trigonométrica seno y coseno con estudiantes de secundaria con edades comprendidas entre los 16 y 17 años. Los temas obtenidos del análisis de los datos proporcionan una diversidad de significados, interpretados y estructurados por categorías semánticas. Estos significados subrayan diferentes interpretaciones del seno y el coseno, de acuerdo con los temas inferidos, como la longitud, la relación, el ángulo y el cálculo de una magnitud. 


\section{CAPÍTULO 3}

\section{METODOLOGÍA DE INVESTIGACIÓN}

En este apartado vamos a detallar la metodología empleada en esta investigación. En primer lugar, caracterizaremos la investigación, continuaremos con las características de la muestra de sujetos participantes en el estudio, detallaremos el proceso de elaboración de los instrumentos de recogida de datos y su procedimiento de aplicación, y finalizaremos con el procedimiento seguido en el análisis de datos.

\subsection{Tipo de estudio y descripción general.}

Esta investigación posee una naturaleza de tipo exploratorio-descriptivo. En primer lugar, se considera exploratorio ya que se realiza con el objetivo de examinar un tema de investigación poco estudiado, las aplicaciones de la tecnología en la resolución de un problema de trigonometría con estudiantes de $1^{\circ}$ bachillerato. En segundo lugar, se considera descriptivo ya que se ha planificado con la finalidad de recoger información para describir y especificar propiedades y características de los distintos significados mostrados por el alumnado sobre la razón trigonométrica tangente mediante la resolución de un problema trigonométrico de forma escrita y mediante el applet. Además, ello permitirá establecer relaciones entre el uso de la representación simbólica y la representación gráfica dada por el applet en la resolución del problema trigonométrico propuesto.

El instrumento de recogida de datos consta de dos cuestionarios. El primer cuestionario constaba de una serie de tareas que detallaremos a continuación. Tras la detección del uso inesperado del applet por parte del alumnado, lo cual nos informó de que no era intuitivo, se determinó la realización de un segundo cuestionario con instrucciones de forma más pautada con la finalidad de profundizar en la interpretación gráfica del problema haciendo uso del applet.

Respecto al análisis de los datos, se ha llevado a cabo una categorización de respuestas, observando los vínculos entre las representaciones verbal y gráfica. A continuación, se han analizado las respuestas con un enfoque cualitativo para interpretar conjuntamente las tareas analizadas. 


\subsection{Sujetos de la investigación y variables.}

La muestra de este estudio está compuesta por 8 estudiantes elegidos de forma intencional y por disponibilidad, formada por 7 mujeres y 1 hombre, del primer curso de bachillerato de la modalidad científico-tecnológica con edades comprendidas entre los 16 y 17 años, matriculados durante el curso académico 2019-2020 en la asignatura Matemáticas I, en un centro de Educación Secundaria Obligatoria de la provincia de Huelva.

Este centro se encuentra en un entorno rural del Condado de Huelva y su población es de aproximadamente 22.000 habitantes. La principal actividad económica es la agricultura, dedicada principalmente al cultivo del fresón y la frambuesa, así como la construcción y el sector servicio y el nivel socioeconómico de las familias es medio. La oferta educativa de este centro es Educación Secundaria Obligatoria y Bachillerato y el número de alumnos/as ronda los 650-700. Además, el centro es Plurilingüe en inglés y francés, es centro TIC y forma parte de multitud de planes educativos entre los que destaca Escuela Espacio de Paz, Plan de Fomento a la Lectura y Plan PROA.

Los estudiantes habían finalizado la unidad de trigonometría varias semanas atrás en el momento de la aplicación de los cuestionarios, por lo que el alumnado tenía conocimientos sobre: la medida de un ángulo en radianes; las razones trigonométricas de un ángulo cualquiera; las razones trigonométricas de los ángulos suma, diferencia de otros dos, doble y mitad; las fórmulas de transformaciones trigonométricas; los Teoremas del Coseno y del Seno; la resolución de ecuaciones trigonométricas sencillas y la resolución de triángulos y de problemas geométricos diversos. Por otro lado, es importante también tener en consideración que los estudiantes ya tenían nociones de trigonometría previas del curso anterior.

\subsection{Elaboración del instrumento de recogida de información.}

El instrumento de recogida de datos fue un cuestionario de respuesta abierta elaborado expresamente para este estudio a través de tres fases.

\section{Fase I: Diseño del cuestionario 1.}

Tras una primera entrevista con el tutor del presente Trabajo Fin de Máster, en la que decidimos realizar un estudio de la resolución de un problema trigonométrico con estudiantes de primer curso de bachillerato mediante un applet creado por el tutor, procedimos a la elaboración de un primer cuestionario. 
El problema trigonométrico planteado fue el siguiente:

"Dos observadores ven una torre desde $\alpha=70^{\circ}$ y $\beta=30^{\circ}$ respectivamente. Con el pie de la torre forman $\gamma=60^{\circ}$ y están a una distancia de $8 \mathrm{~m}(\mathrm{~d})$. ¿Cuál es la altura de la torre en metros? La siguiente ilustración muestra un esquema de la situación descrita en el problema."

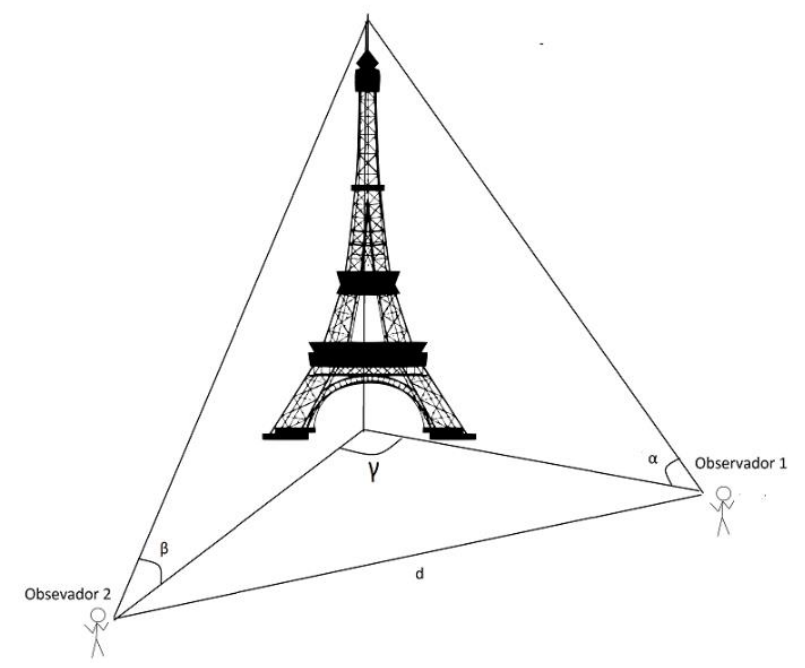

Figura 7. Esquema de la situación descrita en el problema.

En primer lugar, propusimos al alumnado la resolución de forma escrita del problema planteado. El enunciado de la primera cuestión fue la siguiente:

Tarea 1: "Resuelve el problema con ayuda de la calculadora, expresando los cálculos convenientes a mano. Con la intención de que puedas comprobar si tus cálculos han sido apropiados, el applet te facilita la solución 5,06 m, pero nos interesaría la forma en la que has llegado a esa solución."

Con vistas a la consecución de los objetivos propuestos en este trabajo, planteamos las siguientes cuestiones al alumnado tras la observación y manipulación del applet.

Tarea 2. “Podrías explicar tras manipularlo en qué se basa? Explica cómo funciona.”

Tarea 3. "¿Cómo se ha calculado el valor "aproximado” de la altura de la torre a partir de la posición del observador 1? Expresa los cálculos necesarios."

Tarea 4. “CCómo puedes determinar la distancia del observador 2 a la Torre a partir de la distancia del observador 1 a la misma? Expresa los cálculos necesarios.” 
Tarea 5. “¿Cuál es la posición del observador 1 para que la altura aproximada de la torre sea la que nos interesaría calcular, 5,06 m? Expresa los cálculos necesarios.”

Con el fin de determinar si efectivamente los estudiantes habían comprendido el funcionamiento del applet se les propusieron los siguientes problemas a resolver mediante el uso del mismo.

Problema a: "Dos observadores ven una torre desde $\alpha=60^{\circ}$ y $\beta=30^{\circ}$ respectivamente. Con el pie de la torre forman $\gamma=0^{\circ}$ y están a una distancia de $8 \mathrm{~m}$ (d). ¿Cuál es la altura de la torre en metros?"

Problema b: "Dos observadores ven una torre desde $\alpha=60^{\circ}$ y $\beta=30^{\circ}$ respectivamente. Con el pie de la torre forman $\gamma=180^{\circ}$ y están a una distancia de $8 \mathrm{~m} \mathrm{(d).} \mathrm{¿Cuál} \mathrm{es} \mathrm{la}$ altura de la torre en metros?"

Problema c: "Dos observadores ven una torre desde $\alpha=60^{\circ}$ y $\beta=30^{\circ}$ respectivamente. Con el pie de la torre forman $\gamma=45^{\circ}$ y están a una distancia de $8 \mathrm{~m}(\mathrm{~d})$. ¿Cuál es la altura de la torre en metros?"

En todo momento se mantuvieron constantes los ángulos $\alpha$ y $\beta$ y la distancia de separación entre los observadores, $d$, modificando únicamente el ángulo $\gamma$ que forman los dos observadores con el pie de la torre. De esta forma se podía apreciar a simple vista en el applet cómo se modificaba la recta que determina el ángulo entre ambos observadores. No tiene sentido modificar el resto de ángulos puesto que el applet muestra solo la vista superior de la situación y los ángulos $\alpha$ y $\beta$ se encuentran en otra perspectiva.

\section{Fase II: Revisión tras la implementación del primer cuestionario.}

Tras la implementación de este cuestionario los resultados no fueron los esperados ya que detectamos ciertas inconsistencias en el uso del applet por parte del alumnado, por ello procedimos a la realización de un segundo cuestionario introduciendo la información de forma pautada y con un protocolo guiado para emplear el applet y responder cuestiones a partir de su manipulación.

Las cuestiones en las que encontramos errores de comprensión fueron las Tareas 2, 3 y 4. En la Tarea 2, en el que el alumnado debía explicar el funcionamiento del applet, estos hacen referencia principalmente a la resolución manual del problema y no explican en qué se basa el funcionamiento de la aplicación. 
En la Tarea 3, en el que se les pedía un valor "aproximado" de la altura de la torre a partir de la posición del observador 1, el alumnado hacía uso de la razón trigonométrica tangente para el cálculo aproximado de la torre, aunque hacían referencia a que era imposible determinar dicha altura ya que se desconocía el valor de la distancia del observador 1 a la Torre. El alumnado fue incapaz de establecer una relación entre el cálculo algebraico y el cálculo numérico ya que para ellos la distancia del observador 1 a la Torre es una incógnita y no una variable. Ello viene a mostrar las dificultades que presenta el alumnado para trabajar en un ambiente diferente al habitual con medios tecnológicos.

En la Tarea 4, en el que el alumnado debía determinar la distancia del observador 2 a la Torre a partir de la distancia del observador 1 a la misma, estos usan en su mayoría el Teorema del Coseno, del Seno e incluso, erróneamente, la razón trigonométrica coseno. En ninguna de las expresiones anteriores existía una relación directa entre la distancia del observador 1 a la Torre y la distancia del observador 2 a la misma que pedíamos. El alumnado no vio la dependencia entre la distancia del observador 1 y el observador 2 a la Torre. Ello viene a mostrar que el alumnado no percibió la relación directa entre la distancia del observador 1 y el observador 2 que se establece en el applet.

Por otro lado, pudimos comprobar mediante la Tarea 1 que el alumnado dominaba con fluidez la resolución algebraica del problema ya que todos consiguieron resolverlo correctamente. También pudimos ver en la Tarea 5, en que se les pedía cuál era la posición del observador 1 para que la altura aproximada de la torre fuera 5,06 m, que el alumnado realizaba un uso correcto de la razón trigonométrica tangente llegando en todos los casos a una correcta resolución, aunque no era el objetivo perseguido con esta cuestión, que era percibir que justo en esa posición del observador 1, el observador 2 pertenece justamente a la circunferencia de centro observador 1 y radio la distancia entre los observadores.

Por último, respecto a la resolución de los problemas propuestos mediante el uso del applet, pudimos comprobar que el alumnado no había entendido el funcionamiento de la misma ya que se limitaron a la introducción en la aplicación de los ángulos dados y de la distancia entre los observadores, sin percatarse de que el observador 1 podía moverse a lo largo del eje $\mathrm{X}$ y así obtener una altura aproximada a la altura teórica mediante el cálculo iterativo que realiza el applet. Tampoco fueron conscientes de que el observador 2 no se encontraba en la circunferencia que establece las condiciones del problema real. 


\section{Fase III: Diseño del cuestionario 2.}

En este caso, tras la introducción de unas pautas más concretas y directas que ayudaran a la profundización de la interpretación gráfica del problema mediante el applet les planteamos las cuestiones siguientes incluyendo en ellas algunas recomendaciones:

Tarea 1: "Una vez que has introducido los datos del problema en los huecos facilitados, fíjate que el observador 1 puedes moverlo libremente a lo largo del eje X y variar su distancia a la Torre ubicada en el punto A. Observa que la altura de la torre también varía, es decir, estás tomando una medida aproximada de la torre que es diferente a la real que ya la has calculado, porque el observador 1 no está en la posición adecuada. Explica cómo calcular a mano la altura de la torre aproximada en función de la distancia del observador 1 a la misma."

Tarea 2: "Observa que cuando mueves el observador 1, el observador 2 va variando su distancia al punto A. Explica cómo calcular a mano la distancia del observador 2 a la torre en función de la distancia del observador 1 a la misma."

Tarea 3: "Fíjate por último en la circunferencia de centro el observador 1 , ¿De qué radio es esa circunferencia?; ¿Dónde se sitúa el observador 2 cuando la altura calculada es exactamente la real obtenida en la resolución a mano del problema?"

Tarea 4: "Por ello, os propongo que completéis las tablas siguientes y que calculéis la distancia del observador 2 a la torre y la altura de la torre a partir de diferentes distancias del observador 1 a la misma para el caso inicial, es decir, el caso en el que dos observadores ven una torre desde $\alpha=70^{\circ}$ y $\beta=30^{\circ}$ respectivamente. Con el pie de la torre forman $\gamma=60^{\circ}$ y están a una distancia de $8 \mathrm{~m}(\mathrm{~d}) . ”$

\begin{tabular}{|l|l|}
\hline $\mathrm{d}_{1}$ (Distancia del observador 1 a la Torre) & $\mathrm{d}_{2}$ (Distancia del observador 2 a la Torre) \\
\hline $2 \mathrm{~m}$ & \\
\hline $4 \mathrm{~m}$ & \\
\hline $8 \mathrm{~m}$ & \\
\hline
\end{tabular}

\begin{tabular}{|l|l|}
\hline $\mathrm{d}_{1}$ (Distancia del observador 1 a la Torre) & $\begin{array}{l}\mathrm{h} \text { (Altura de la torre vista por el } \\
\text { observador } 1 \text { con ángulo fijo } \alpha)\end{array}$ \\
\hline $2 \mathrm{~m}$ & \\
\hline $4 \mathrm{~m}$ & \\
\hline $8 \mathrm{~m}$ & \\
\hline
\end{tabular}


Por último, se les propuso resolver con el applet los tres problemas propuestos al final del cuestionario 1 cuyos enunciados mostramos a continuación.

Problema a: "Dos observadores ven una torre desde $\alpha=60^{\circ}$ y $\beta=30^{\circ}$ respectivamente. Con el pie de la torre forman $\gamma=0^{\circ}$ y están a una distancia de $8 \mathrm{~m}$ (d). ¿Cuál es la altura de la torre en metros?"

Problema b: "Dos observadores ven una torre desde $\alpha=60^{\circ}$ y $\beta=30^{\circ}$ respectivamente. Con el pie de la torre forman $\gamma=180^{\circ}$ y están a una distancia de $8 \mathrm{~m}$ (d). ¿Cuál es la altura de la torre en metros?"

Problema c: "Dos observadores ven una torre desde $\alpha=60^{\circ}$ y $\beta=30^{\circ}$ respectivamente. Con el pie de la torre forman $\gamma=45^{\circ}$ y están a una distancia de $8 \mathrm{~m}$ (d). ¿Cuál es la altura de la torre en metros?"

\subsection{Procedimiento de aplicación del instrumento.}

Para la realización de esta investigación se implementaron dos cuestionarios. El primer cuestionario se aplicó a un total de 8 alumnos, concretamente 7 alumnas y 1 alumno y el segundo cuestionario se aplicó solamente a 5 alumnos, concretamente, 4 alumnas y 1 alumno. En primer lugar, se implementó un primer cuestionario y tras la revisión de las respuestas del alumnado, decidimos implementar el segundo cuestionario para profundizar en la interpretación gráfica del problema haciendo uso del applet.

El primer cuestionario constaba de 5 tareas y de 3 problemas similares al problema inicial propuesto. Este primer cuestionario fue enviado al alumnado en PDF a través del correo electrónico el día 13 de Abril de 2020, siendo devueltos completados el 19 de Abril de 2020 en formato PDF. Este cuestionario no se realizó de forma anónima porque además de las respuestas debieron incluir sus datos de identificación: nombre y apellidos y curso.

El segundo cuestionario constaba de 4 tareas y de los 3 problemas del cuestionario 1. Este segundo cuestionario fue enviado al alumnado en PDF a través del correo electrónico el día 22 de Abril de 2020, siendo devueltos completados el 3 de Mayo de 2020 en formato PDF. Igual que en el cuestionario anterior, además de las respuestas debieron incluir sus datos de identificación.

En principio, la idea era realizar un taller con el alumnado con el fin de tratar el problema inicial propuesto antes de la realización del primer cuestionario. Dada la situación 
sanitaria surgida nos vimos obligados a adaptar e implementar los cuestionarios de esta forma.

\subsection{Tipo de análisis realizado.}

Para el análisis de las producciones del alumnado se ha llevado a cabo un análisis del contenido, el cual se realiza mediante la descomposición de las respuestas en sus unidades más simples, a partir de las cuales se sintetizan de modo sistemático unos temas y se identifican unas categorías (Rico, 2013).

Este análisis es de tipo cualitativo, procediendo a realizar una codificación, en la que se consideran segmentos o unidades simples de contenido que identifican hechos, conceptos y destrezas, a partir de los cuales se inducen categorías mediante su análisis y comparación.

Además, se trata de un proceso inductivo, en el cual las categorías, relaciones y modelos aparecen a partir de los datos. Se trata también de un proceso cíclico en la medida que forma parte de todas las etapas de la investigación (McMillan y Schumacher, 2005).

El análisis tiene lugar en cuatro etapas:

- Primera etapa: Organizar los datos en temas.

- Segunda etapa: Desarrollar los temas en categorías.

- Tercera etapa: Analizar las categorías: semejanzas, diferencias y vinculaciones.

- Cuarta etapa: Interpretar los datos mediante las categorías y mostrar los resultados inferidos. 


\section{CAPÍTULO 4}

\section{ANÁLISIS DE LOS DATOS}

En este capítulo se presenta la descripción y la interpretación de los datos aportados por los cuestionarios. En primer lugar, se describe el proceso de análisis realizado. A continuación, se muestra el análisis general de las respuestas del cuestionario 1 realizado por los 8 estudiantes.

Por último, se muestra el análisis de casos de las respuestas del cuestionario 2, analizando las tareas y los problemas planteados de forma detallada para 5 de los estudiantes que previamente habían respondido al primer cuestionario.

\subsection{Proceso de análisis.}

Tras la implementación del instrumento, se procedió a realizar el análisis de los cuestionarios teniendo en cuenta tanto las respuestas escritas como las representaciones gráficas de las producciones del alumnado.

El análisis realizado es de tipo cualitativo y para ello, en primer lugar, se realizó una primera lectura y una observación cuidadosa de las producciones del alumnado para identificar y recoger todas aquellas expresiones, representaciones, ideas y conceptos relevantes que se presentan en las mismas. De esta forma, aparecieron de manera natural y sistemática unos primeros temas en las producciones de los estudiantes, que identificaban las unidades de información, con las que se reducían y organizaban la diversidad de respuestas obtenidas.

En una segunda fase de análisis, se organizaron los temas en categorías y subcategorías, las cuales se construyeron, observando y comparando las distintas respuestas en cada uno de los temas y las propiedades relevantes que expresaban, identificando semejanzas y diferencias entre ellas (Coleman y Unrau, 2005; Baptista, Fernández y Sampieri, 2010).

En una tercera fase de análisis, realizamos el análisis de la categoría. Se identificaron semejanzas y diferencias entre ellas y se buscaron posibles relaciones de vinculación o de subordinación entre categorías. El objetivo era obtener patrones que mostraran la estructura de las respuestas e interpretaran los temas y las categorías previamente establecidas. 
Por último, se llevó a cabo la interpretación de los datos mediante su descripción, la presencia de cada categoría y sus relaciones. De esta forma, teniendo en cuenta esos temas, categorías y relaciones, desarrollamos interpretaciones que muestran los resultados de la investigación (Baptista, Fernández y Sampieri, 2010).

\subsection{Análisis general de las respuestas del cuestionario 1.}

A continuación, mostramos el análisis de las respuestas del cuestionario 1, analizando cada uno de las tareas de forma detallada.

Tarea 1: "Resuelve el problema con ayuda de la calculadora, expresando los cálculos convenientes a mano. Con la intención de que puedas comprobar si tus cálculos han sido apropiados, el applet te facilita la solución $5,06 \mathrm{~m}$, pero nos interesaría la forma en la que has llegado a esa solución."

Los temas principales que se encuentran en las respuestas del alumnado para esta tarea lo forman el uso de esquema y el lenguaje algebraico.

Al organizar los datos relativos al tema "uso de esquema" se encontraron 5 unidades de análisis: los elementos no relevantes del problema, los triángulos representados, los ángulos representados, las incógnitas marcadas sobre los lados de los diferentes triángulos y la distancia entre los dos observadores.

Para la unidad de análisis de elementos no relevantes, observamos la presencia de monigotes o figuras que simulaban a los observadores y que no son características estructurales del problema, así como la presencia de ángulos no dados en el problema y que no son necesarios para resolver el problema.

Para la unidad de análisis triángulo, observamos que había alumnos que identificaban la presencia de triángulos rectángulos y lo señalizaban con el símbolo del ángulo recto y otros que, aún identificados o asumidos, no lo señalizaron.

Para la unidad de análisis ángulo, observamos que había alumnos que marcaban los ángulos interiores agudos con un arco indicando así su amplitud y el ángulo interior recto con el símbolo del ángulo recto.

Para la unidad de análisis incógnitas marcadas sobre los lados de los diferentes triángulos, había alumnos que marcaron las incógnitas del problema ( $\mathrm{a}, \mathrm{b}$ y h) y otros que además 
marcaban, de forma innecesaria, como incógnitas ( $\mathrm{X}$ e $\mathrm{Y}$ ) las hipotenusas de los triángulos rectángulos.

Para la unidad de análisis distancia entre los dos observadores, había alumnos que indicaban directamente la distancia concreta entre los observadores $(8 \mathrm{~m})$ y otros que la indicaban de forma simbólica mediante la letra $d$.

A continuación, en la Figura 8 se muestra algunos de los esquemas usados por los estudiantes:

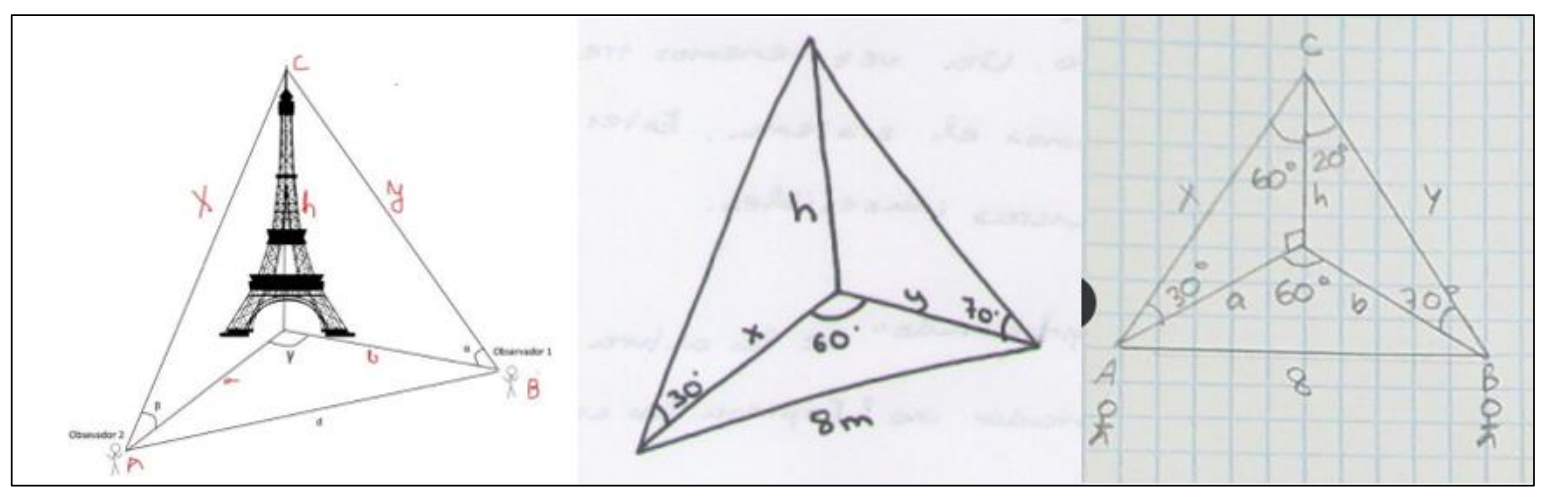

Figura 8. Esquemas empleados por el alumnado en la Tarea 1.

Sobre el tema "lenguaje algebraico" no existen diferencias en el alumnado ya que éstos emplean un método de resolución estandarizado y cuadriculado. Por ello, se muestra en la Figura 9 un ejemplo de la resolución empleada ya que el 100\% del alumnado empleó este método.

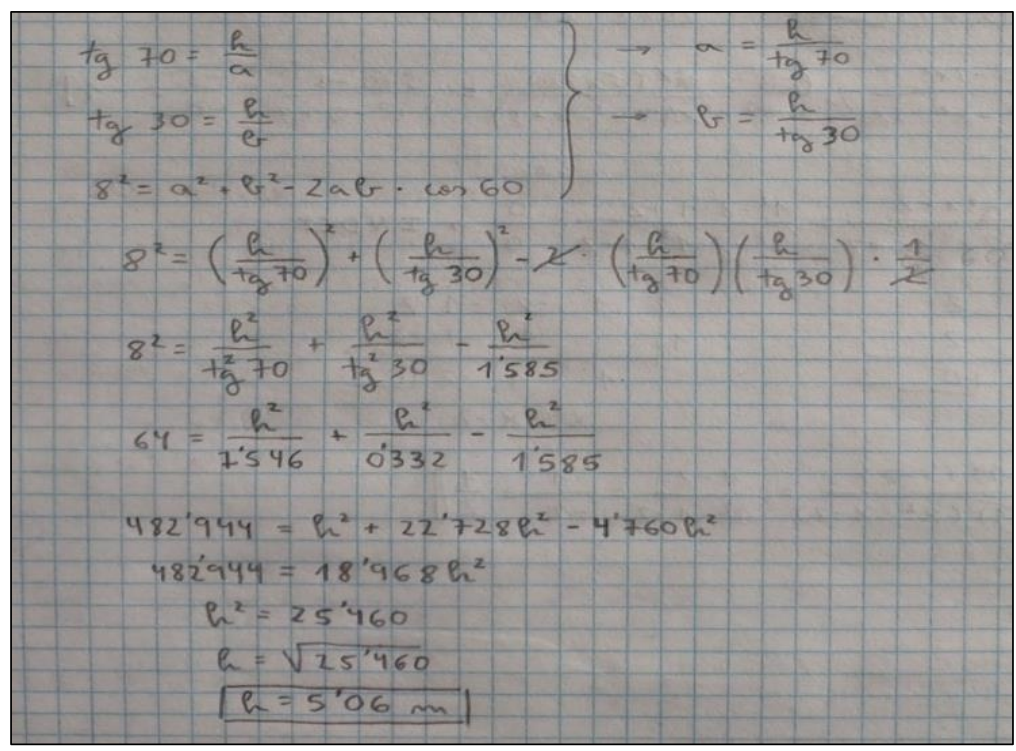

Figura 9. Ejemplo de resolución algebraica empleada por el alumnado en la Tarea 1. 


\section{Tarea 2. “¿Podrías explicar tras manipularlo en qué se basa? Explica cómo funciona."}

A continuación, mostramos algunos fragmentos relevantes del análisis de las respuestas del alumnado para esta tarea.

\section{Fragmento 1:}

Es un problema que trabaja con la trigonometría y para resolverlo he utilizado las tangentes de los dos ángulos con los que cada observador ve la torre y el Teorema del Coseno con el ángulo que forman los dos observadores con el pie de la torre. Con esas tres ecuaciones he creado un sistema.

En este caso el alumno no responde a lo que se pide, directamente resume el proceso de resolución, pero no da ninguna indicación sobre lo que el applet aporta a la misma.

\section{Fragmento 2:}

La aplicación te muestra que al cambiar la posición del observador 1 deben de cambiar las del 2 ya que todos los ángulos se mantienen constantes y así los triángulos son semejantes.

Esta aportación resulta muy interesante, pero no es eficaz para abordar la auténtica finalidad del applet. La alumna ha atribuido un uso alternativo al mismo, que busca trabajar otro contenido relacionado como es la semejanza de triángulos.

\section{Fragmento 3:}

Sí, funciona tal que como todos los ángulos permanecen constantes, al cambiar la posición del observador 1, deben cambiar las del observador dos, para que los triángulos sean semejantes.

En este caso también se muestra que el applet se basa en la semejanza de triángulos, pero no llega a establecer cómo usar el applet para estimar la altura de la torre.

\section{Fragmento 4:}

Por lo que he visto, supongo que esta plataforma resuelve el problema a través de un eje de coordenadas. Sobre este eje coloca en un punto del eje X al observador 1 y traza una recta que forme un ángulo con respecto al eje $X$ igual al ángulo que forman los observadores con el pie de la torre. En esa misma recta coloca, a la distancia propuesta 
en el enunciado, al observador 2. Por último, traza una circunferencia de radio igual a la distancia que separa ambos observadores, cuyo centro es el punto en el que se encuentra el observador 1.

En este caso la alumna simplemente destaca aspectos superficiales del applet y no su funcionamiento interno. No expresa que la posición solución del observador 1 será aquella en la que el observador 2 esté en la circunferencia de centro observador 1 y radio d, por tanto, se queda en aspectos superficiales. Tampoco expresa cómo se genera $\mathrm{d}_{2}$ a partir de $\mathrm{d}_{1} \mathrm{y} h$.

Con base en los fragmentos anteriores se pueden establecer 3 categorías de respuesta:

- Los que enfatizan la resolución algebraica del problema sin identificar la potencialidad del applet como estimador y obtención dinámica de la solución. El grado de instrumentalización del artefacto es por tanto bajo.

- Los que dotan de uso alternativo al applet, pero no es eficaz para abordar la auténtica finalidad de la aplicación. Se produce una instrumentalización del artefacto, pero no coherente con su uso esperado.

- Los que destacan aspectos superficiales del applet y no las relaciones matemáticas que se establecen en el mismo. El grado de instrumentalización del artefacto es bajo, al no reflejar las relaciones matemáticas profundas que regulan su funcionamiento.

Tarea 3. “Cómo se ha calculado el valor "aproximado” de la altura de la torre a partir de la posición del observador 1? Expresa los cálculos necesarios."

A continuación, mostramos algunos fragmentos relevantes del análisis de las respuestas del alumnado para esta tarea.

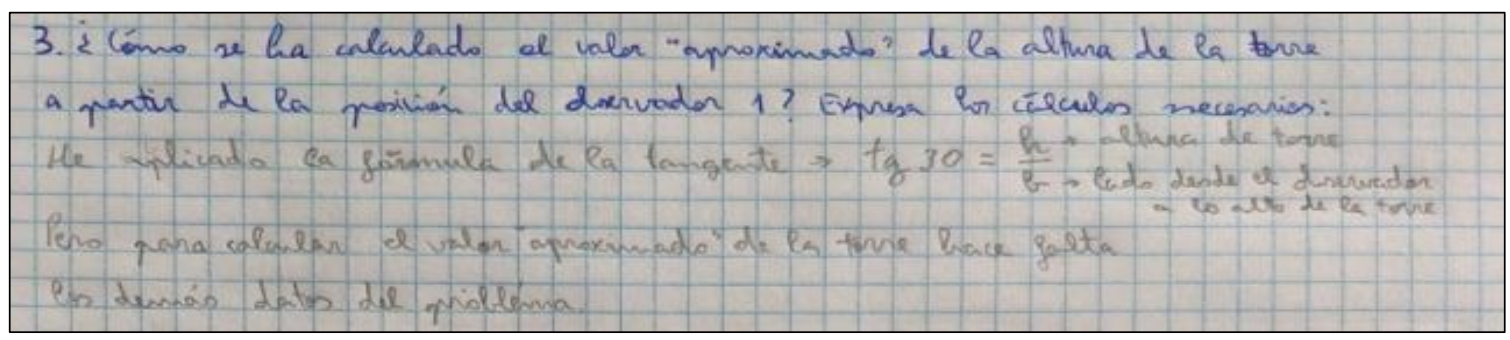

Figura 10. Fragmento de la respuesta de un alumno a la Tarea 3.

En este caso el alumno toma el ángulo de visión del observador 2 y averigua la tangente, pero observa que tiene dos incógnitas y no puede despejarlas. Ello pone de manifiesto 
que el alumno no percibe que el observador 1 es "manipulable" y que la altura iterativa se determina directamente con la operación $h=\operatorname{tg}(\alpha) \cdot d_{1}$.

Además, el alumno informa que desconoce el valor de $\mathrm{d}_{1} \mathrm{y}$ que no se puede averiguar sin un sistema de ecuaciones. La gran utilidad del applet es que a pesar de que la distancia $\mathrm{d}_{1}$ es un valor oculto al estudiante, al no apreciarse con exactitud en la recta numérica, este es utilizado por el applet para calcular la altura iterativa que se les muestra como resultado dependiente del parámetro $\mathrm{d}_{1}$, que funciona como variable en lugar de incógnita.

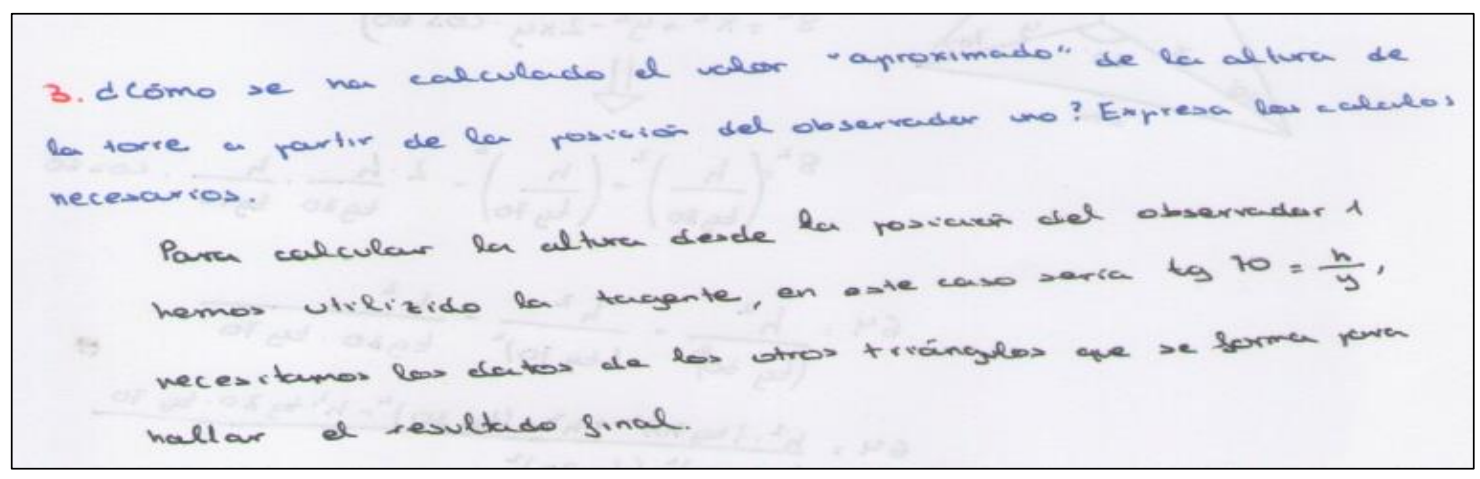

Figura 11. Fragmento de la respuesta de una alumna a la Tarea 3.

En este caso la alumna también manifiesta una inconsistencia en la falta de comprensión de la letra como valor variable ( $\mathrm{d}_{1}$, en este caso expresada como $y$ ). Esta alumna considera la letra como incógnita y no como parámetro/variable.

Señalamos otro fragmento, proporcionado por otra alumna:

Con los datos de los que disponemos resultaría imposible calcular la altura de la torre a partir de la posición del observador 1, ya que solo nos proporcionan el ángulo de vista a la torre, y con solo este dato no podríamos resolver la ecuación, que tendría más de una incógnita. Para poder averiguar la altura de la torre es necesario hacerlo con un sistema de ecuaciones.

Esta alumna también establece la relación esperada, pero parece seguir enfatizando que hace falta averiguar $d_{1}$, cuando el applet lo usa como parámetro y pueden ver una aproximación de $\mathrm{d}_{1}$ en el applet (por la escala gráfica), aunque desconozcan su valor exacto.

Tras el análisis de las respuestas del alumnado sobre esta tarea hemos apreciado que la totalidad de los estudiantes parte de la altura teórica de la torre, para calcular la distancia del observador 1 asociada, no el proceso inverso, que es el objetivo de esta tarea. 


\section{Tarea 4. “Cómo puedes determinar la distancia del observador 2 a la Torre a partir}

de la distancia del observador 1 a la misma? Expresa los cálculos necesarios."

A continuación, mostramos algunos fragmentos relevantes del análisis de las respuestas del alumnado para esta tarea.

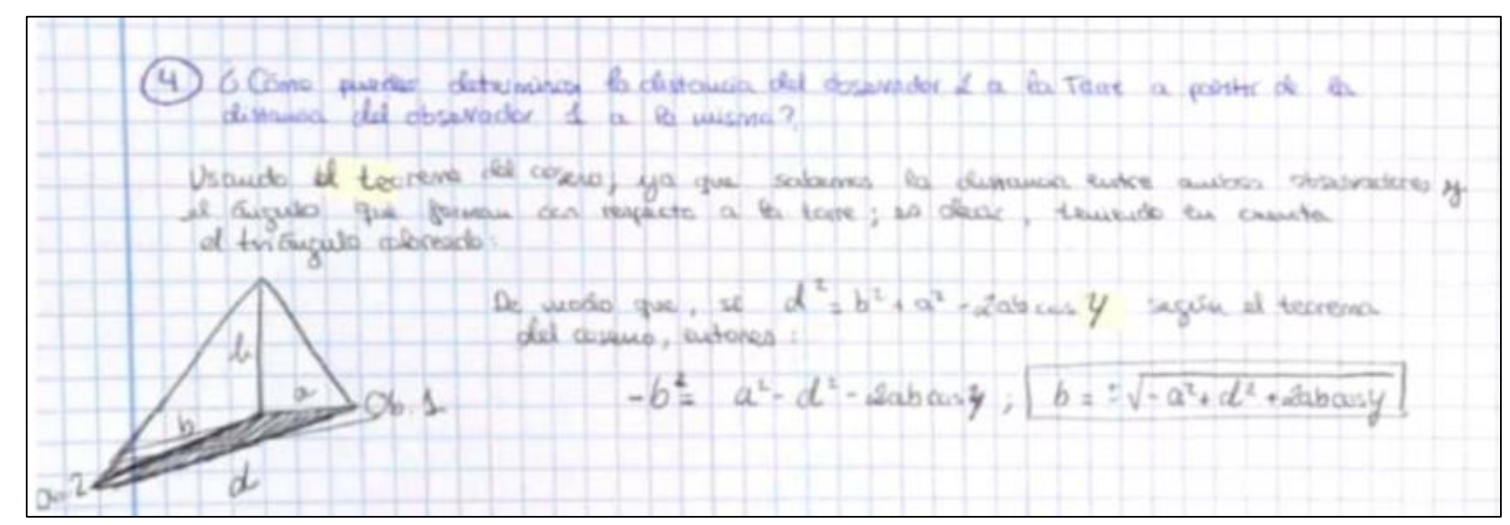

Figura 12. Respuesta de una alumna a la Tarea 4 empleando el Teorema del Coseno.

En este caso la alumna afirma que como $\mathrm{d}$ y $\mathrm{d}_{1}$ son datos conocidos puede aplicar el Teorema del Coseno para calcular $\mathrm{d}_{2}$. De esta forma, está imponiendo que la distancia $\mathrm{d}$ es fija y vale $8 \mathrm{~m}$, cuando en realidad es variable conforme movemos $\mathrm{d}_{1}$. La potencia del applet reside en que se puede estimar la distancia de $\mathrm{d}_{2}$, sin necesidad de imponer la distancia entre observadores, solo manteniendo los ángulos de visión invariantes.

Otro fragmento:

En primer lugar, debo calcular la distancia del observador 1 hacia la torre, lo cual puedo hacer mediante la tangente. Una vez conozco la distancia a la que se encuentran ambos observadores, la distancia a la que se encuentra el observador 1 de la torre, y el ángulo que forman con el pie de la torre; puedo usar el Teorema del Coseno para calcular la distancia del observador 2 a la torre, de la siguiente manera: 


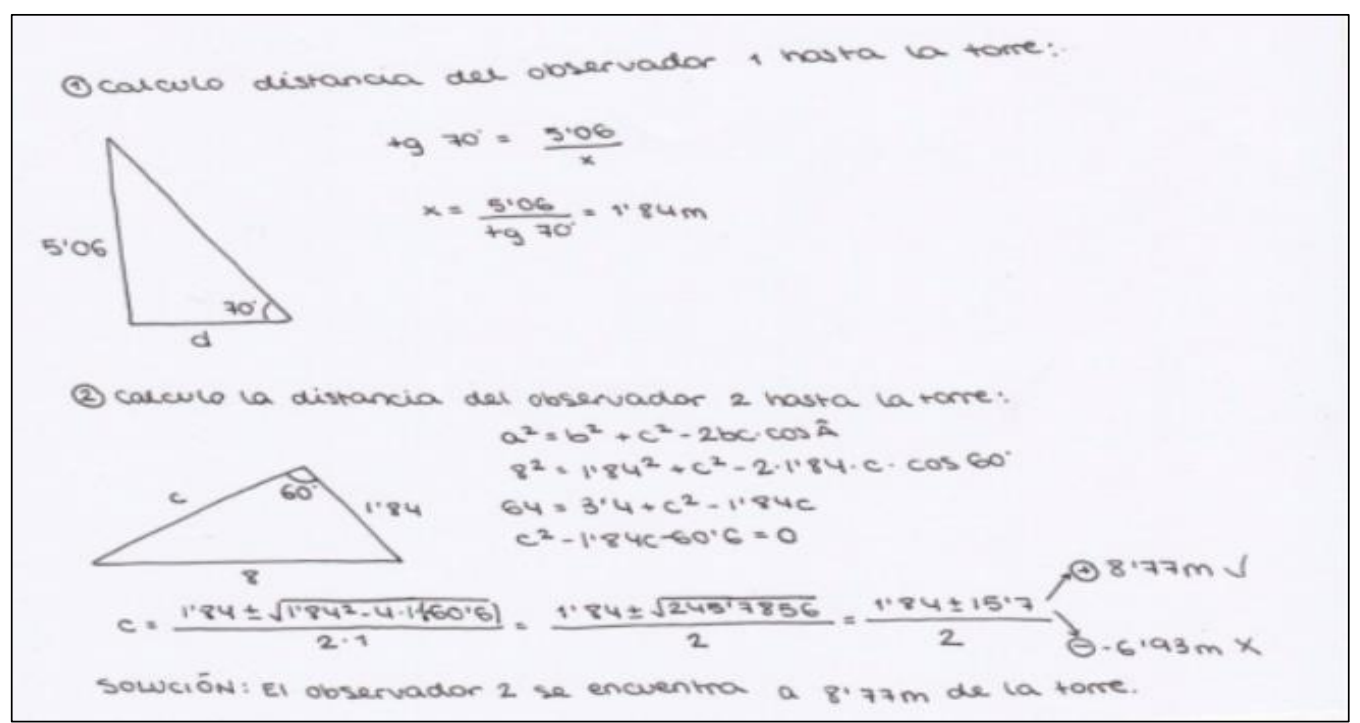

Figura 13. Respuesta de una alumna a la Tarea 4 partiendo del problema resuelto.

En este caso la alumna parte del problema resuelto. A partir de la altura teórica h, calcula $\mathrm{d}_{1}$, y mediante el Teorema del Coseno calcula el valor de $d_{2}$. En realidad, la relación que usa el applet es $\mathrm{d}_{2}=\mathrm{d}_{1} \cdot \frac{\operatorname{tg}(\alpha)}{\operatorname{tg}(\beta)}$, esta relación no depende de la distancia entre observadores, sino de los ángulos de visión que permanecen invariantes.

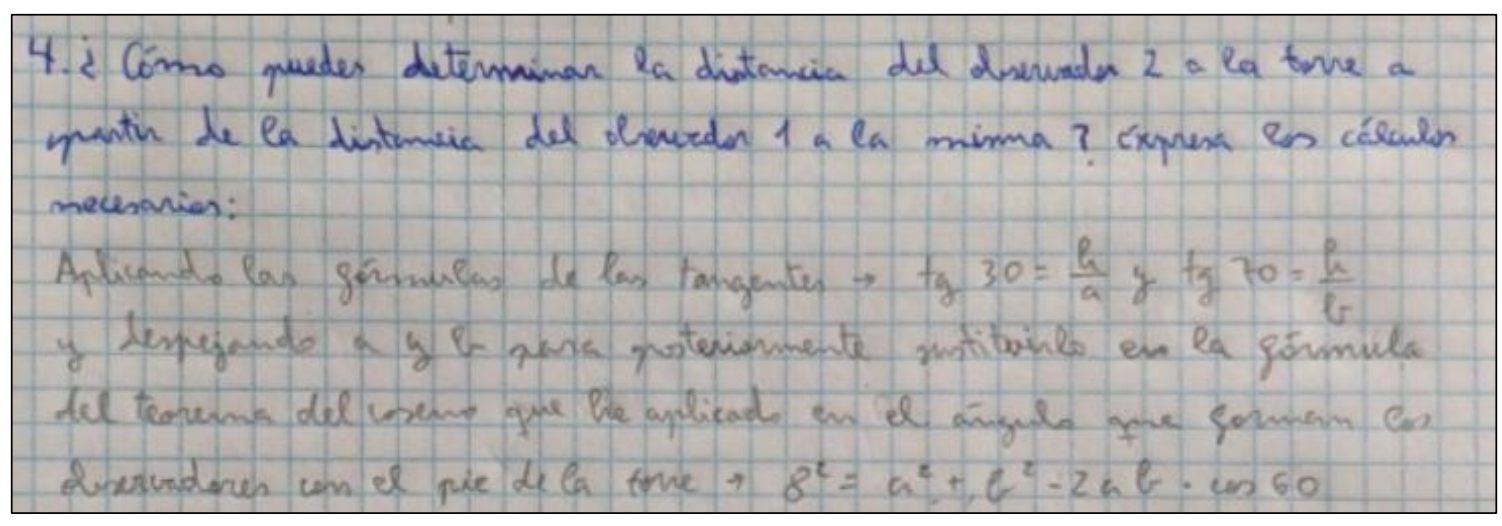

Figura 14. Respuesta de un alumno a la Tarea partiendo de las relaciones de $\mathrm{d}_{1} \mathrm{y} \mathrm{d}_{2}$.

En este caso, el alumno parte de las relaciones de $\mathrm{d}_{1} \mathrm{y} \mathrm{d}_{2}$ con la altura teórica, y despeja en función de h, para sustituir en la ecuación del Teorema del Coseno, pero no establece una relación directa entre $\mathrm{d}_{1} \mathrm{y} \mathrm{d}_{2}$, obtenida al poner la altura teórica de la torre $(\mathrm{h})$ en función de $d_{1}$. 


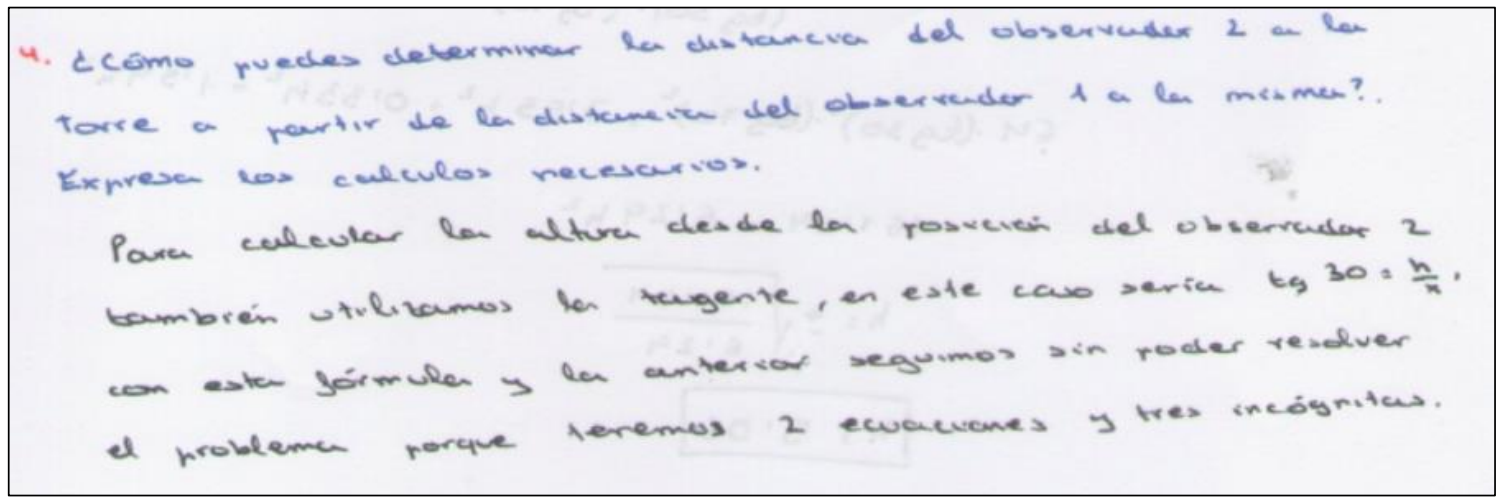

Figura 15. Respuesta de una alumna a la Tarea 4 que establece la relación directamente con la altura teórica de la torre.

En este caso la alumna establece la relación directamente con la altura teórica de la torre (h), sin pasar por $\mathrm{d}_{1}, \operatorname{tg}\left(30^{\circ}\right)=\mathrm{h} / \mathrm{d}_{2}$, que junto con $\operatorname{tg}\left(70^{\circ}\right)=\mathrm{h} / \mathrm{d}_{1}$, obtiene 2 ecuaciones y 3 incógnitas. No considera $\mathrm{d}_{1}$ como variable independiente.

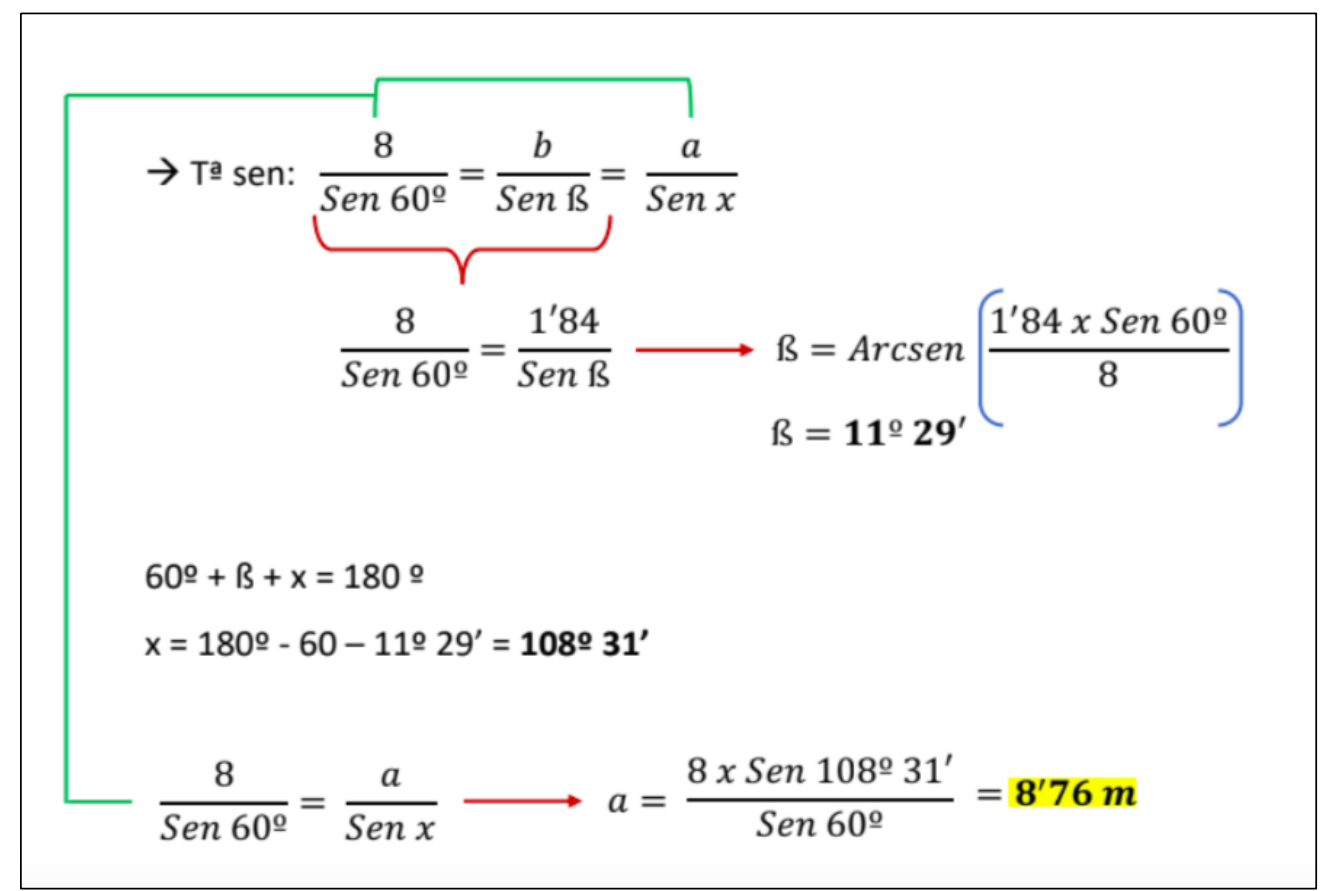

Figura 16. Respuesta de una alumna a la Tarea 4 empleando el Teorema del Seno.

En este otro caso la alumna usa el Teorema del Seno, imponiendo la distancia $\mathrm{d}=8 \mathrm{~m}$ y la solución teórica de $\mathrm{d}_{1}$, para sacar a partir del teorema el valor de $\mathrm{d}_{2}$. 
4. ¿Cómo puedes determinar la distancia del observador 2 a la Torre a partir de la distancia del observador 1 a la misma? Expresa los cálculos necesarios.

\section{Esta vez utilizamos la razón del coseno}

$\operatorname{Cos} 60^{\circ}=\frac{\text { contiguo }}{\text { hipotenusa }}$

$0,5=\frac{\text { distancia del observador } 1 \text { a la torre }}{\text { distancia del observador } 2 \text { a la torre }} \rightarrow$

Distancia del obs 2 a la torre $=\frac{\text { distsncia del observador } 1 \text { a la torre }}{0,5}$

Distancia del obs 2 a la torre $=\frac{1,84}{0,5} \rightarrow 3,68$ metros

Figura 17. Respuesta de una alumna a la Tarea 4 empleando la razón trigonométrica coseno.

En este caso la alumna incurre en el error de considerar que el triángulo que forma los dos observadores con la torre es rectángulo y aplica el coseno de $60^{\circ}$, para sacar $\mathrm{d}_{2}$ a partir del valor teórico para $\mathrm{d}_{1}=1,84 \mathrm{~m}$.

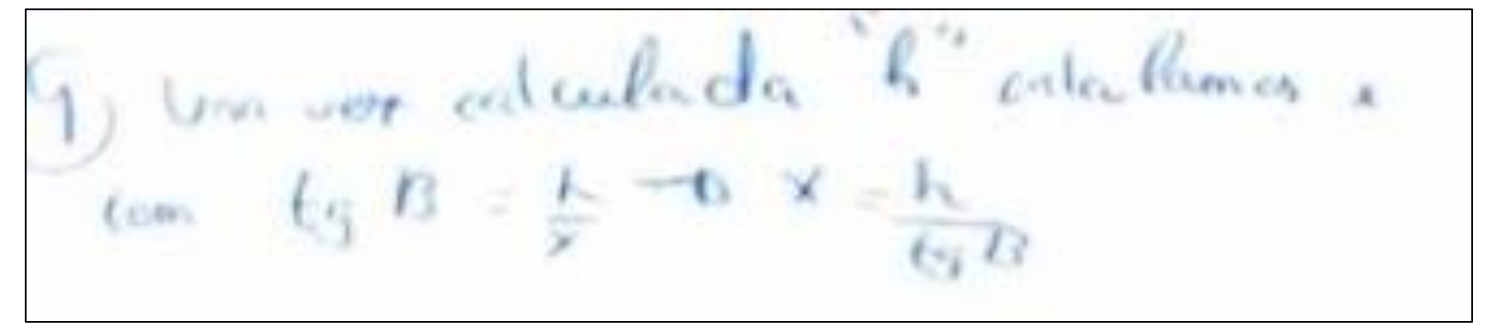

Figura 18. Respuesta de una alumna a la Tarea 4 empleando la relación de $\mathrm{d}_{2}$ con la altura teórica de la torre, pero sin imponer la relación entre h y d $\mathrm{d}_{1}$.

Por último, esta alumna usa la relación entre la altura teórica de la torre (h) y d 2 , pero no impone que h se sustituye por $d_{1} \cdot \operatorname{tg}(\alpha)$.

Con base en los fragmentos anteriores se pueden establecer 3 categorías de respuesta:

- Los que establecen relaciones entre variables, pero no identifican explícitamente el papel del applet en establecerlas. El grado de instrumentalización es bajo.

- Los que consideran que sin datos concretos no se puede calcular $\mathrm{d}_{2}$ porque hace falta resolver una ecuación que, en realidad, expresa una relación funcional entre variables una de las cuales puede ser manipulada. Puede deberse a un obstáculo al interpretar el significado de las letras mediante su uso como incógnita exclusivamente. 
- Los que parten de los resultados solución para establecer relaciones. Por ejemplo, $\mathrm{h}=5,06 \mathrm{~m}$, de donde $\mathrm{d}_{1}=1,84 \mathrm{~m}$, para calcular posteriormente $\mathrm{d}_{2}$. O sabiendo que $\mathrm{d}=8 \mathrm{~m}$ y $\mathrm{d}_{1}=1,84 \mathrm{~m}$, aplican Teorema del Seno o del Coseno, para calcular $\mathrm{d}_{2}$. Esto puede también estar incentivado por haberle proporcionado el valor exacto de la altura en el applet.

Tarea 5. “Cuál es la posición del observador 1 para que la altura aproximada de la torre sea la que nos interesaría calcular, 5,06 m? Expresa los cálculos necesarios."

En este caso, la totalidad de los estudiantes responde que $\mathrm{d}_{1}$ se calcula mediante:

$$
\mathrm{d}_{1}=\frac{5,06}{\operatorname{tg}\left(70^{\circ}\right)}=1,84 \mathrm{~m}
$$

En realidad, lo que se esperaba es que informaran que cuando $\mathrm{d}_{1}=1,84 \mathrm{~m}$, el observador 2 está justo en la circunferencia de centro $\mathrm{O}_{1} \mathrm{y}$ radio $\mathrm{d}=8 \mathrm{~m}$, pero ninguno llegó a establecer esta conexión con el applet.

Esta tarea realmente marca una ruptura patente entre el empleo o comprensión del applet y la resolución algebraica formal del problema, espontáneamente no identifican la utilidad del applet.

Por último, en cuanto a las representaciones gráficas empleadas en los problemas propuestos siguientes:

Problema a: "Dos observadores ven una torre desde $60^{\circ}$ y $30^{\circ}$ respectivamente. Con el pie de la torre forman $0^{\circ}$ y están a una distancia de $8 \mathrm{~m}$ (d). ¿Cuál es la altura de la torre en metros?"

Problema b: "Dos observadores ven una torre desde $60^{\circ}$ y $30^{\circ}$ respectivamente. Con el pie de la torre forman $0^{\circ}$ y están a una distancia de $8 \mathrm{~m}(\mathrm{~d})$. ¿Cuál es la altura de la torre en metros?"

Problema c: "Dos observadores ven una torre desde $60^{\circ}$ y $30^{\circ}$ respectivamente. Con el pie de la torre forman ${ }^{\circ}$ y están a una distancia de $8 \mathrm{~m}(\mathrm{~d})$. ¿Cuál es la altura de la torre en metros?"

La totalidad de los participantes se limitó a introducir los datos en el applet y a realizar una captura de la pantalla de la misma. Ninguno percibió que el observador 1 podía cambiar de posición arbitrariamente. En la Figura 19 que se muestra a continuación puede 
apreciarse una captura de pantalla de la resolución del problema a) realizado por una alumna.

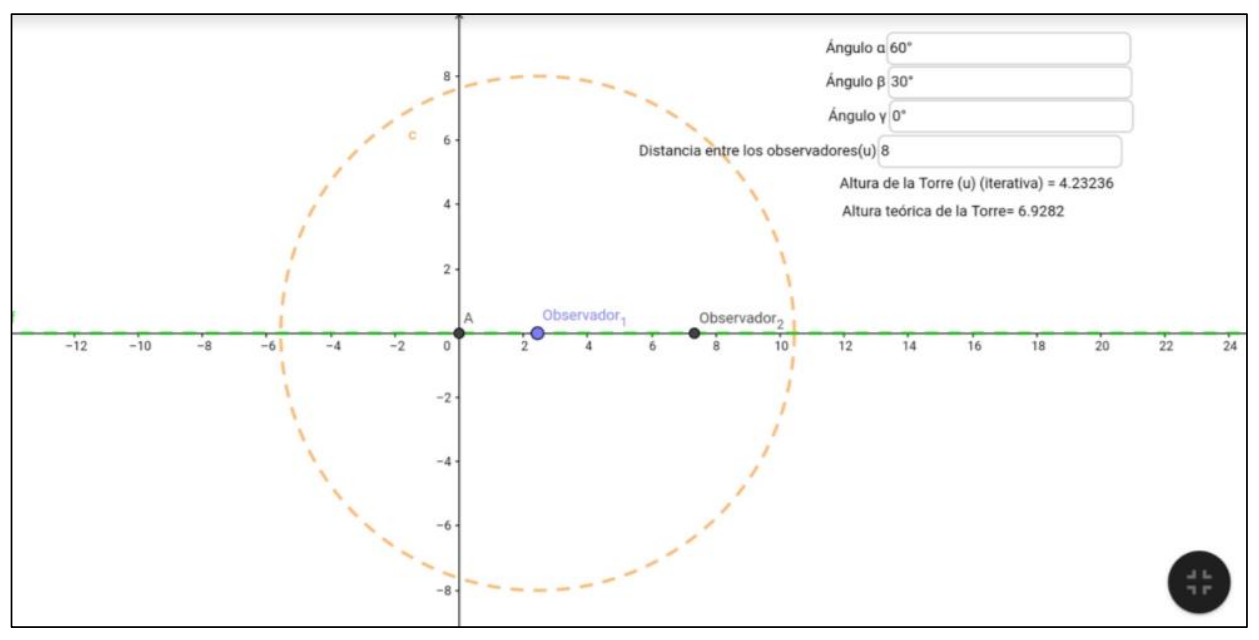

Figura 19. Captura de pantalla del problema a) realizada por una alumna.

\subsection{Estudio de casos de las respuestas del cuestionario 2 .}

A continuación, mostramos el análisis de las respuestas del cuestionario 2, analizando cada una de las tareas y de los problemas planteados de forma detallada para 5 de los alumnos con nombres ficticios: Carlos, Marta, Ana, Laura y María.

Con este tipo de análisis pretendemos analizar la evolución del estudiante a lo largo de todo el cuestionario y en relación con el cuestionario anterior y establecer semejanzas y diferencias con las respuestas de sus compañeros.

La Tarea 4 de este cuestionario se analizará de forma conjunta para los 5 estudiantes ya que de esta manera se puede observar las semejanzas y diferencias respecto a los resultados numéricos obtenidos.

\section{Caso 1: Carlos}

Tarea 1: "Explica cómo calcular a mano la altura de la torre aproximada en función de la distancia del observador 1 a la misma." 
Si llamamos $d_{1}$ a la distancia del observador 1 a la torre, como conocemos el ángulo que forma este y la torre, podemos calcular la altura de la torre aproximada $(h)$ aplicando la fórmula de la tangente:

$$
\operatorname{tg} 70=\frac{h}{d_{1}} ; h=\operatorname{tg} 70 \cdot d_{1}
$$

Figura 20. Respuesta de Carlos a la Tarea 1 expresando la relación entre la altura de la torre y $\mathrm{d}_{1}$ simbólicamente.

En este caso Carlos expresa la relación entre la altura de la torre y $\mathrm{d}_{1}$ empleando exclusivamente el lenguaje simbólico.

Tarea 2: "Explica cómo calcular a mano la distancia del observador 2 a la torre en función de la distancia del observador 1 a la misma."

$$
\begin{aligned}
& \text { Si llamamos } d_{1} \text { a la distancia del observador } 1 \text { a la torre y } d_{2} \text { a la distancia de } \\
& \operatorname{tg} 70=\frac{h}{d_{1}} \quad \mathrm{y} \quad \operatorname{tg} 30=\frac{h}{d_{2}} \\
& \operatorname{tg} 70=\frac{h}{d_{1}} \quad h=\operatorname{tg} 70 \cdot d_{1} \\
& \operatorname{tg} 30=\frac{h}{d_{2}} \quad \rightarrow \quad h=\operatorname{tg} 30 \cdot d_{2} \quad \rightarrow \quad \operatorname{tg} 30 \cdot d_{2}=\operatorname{tg} 70 \cdot d_{1}
\end{aligned}
$$

Figura 21. Respuesta de Carlos a la Tarea 2 expresando la relación entre $\mathrm{d}_{1} \mathrm{y}_{\mathrm{d}}$ simbólicamente.

Carlos deduce simbólicamente la relación esperada en el cuestionario 1: $\operatorname{tg}\left(30^{\circ}\right) \cdot \mathrm{d}_{2}=\operatorname{tg}\left(70^{\circ}\right) \cdot \mathrm{d}_{1}$

Tarea 3: “¿De qué radio es esa circunferencia?; ¿Dónde se sitúa el observador 2 cuando la altura calculada es exactamente la real obtenida en la resolución a mano del problema?" 
Podemos observar que en la circunferencia el observador 1 se encuentra en el centro de esta y que el radio de la circunferencia es la distancia entre el observador 1 y el observador 2 .

Cuando la altura iterativa es exactamente igual a la altura teórica el observador 2 se encuentra en un punto por el que pasa la circunferencia.

Figura 22. Respuesta de Carlos a la Tarea 3 identificando correctamente el radio de la circunferencia y la posición óptima de $\mathrm{O}_{2}$.

Carlos realiza la identificación adecuada del radio de la circunferencia y la posición óptima cuando la altura iterativa coincide con la teórica, en la que $\mathrm{O}_{2}$ está sobre la circunferencia de centro $\mathrm{O}_{1}$ y radio d.

Al final del cuestionario 2 se les propuso resolver con el applet los tres problemas propuestos al final del cuestionario 1 cuyos enunciados mostramos a continuación.

Problema a: "Dos observadores ven una torre desde $60^{\circ}$ y $30^{\circ}$ respectivamente. Con el pie de la torre forman $0^{\circ}$ y están a una distancia de $8 \mathrm{~m}(\mathrm{~d})$. ¿Cuál es la altura de la torre en metros?"

Problema b: "Dos observadores ven una torre desde $60^{\circ}$ y $30^{\circ}$ respectivamente. Con el pie de la torre forman $180^{\circ}$ y están a una distancia de $8 \mathrm{~m}$ (d). ¿Cuál es la altura de la torre en metros?"

Problema c: "Dos observadores ven una torre desde $60^{\circ}$ y $30^{\circ}$ respectivamente. Con el pie de la torre forman $45^{\circ}$ y están a una distancia de $8 \mathrm{~m}(\mathrm{~d})$. ¿Cuál es la altura de la torre en metros?"

Carlos ajusta totalmente los diagramas para que coincidan hasta la precisión establecida. 


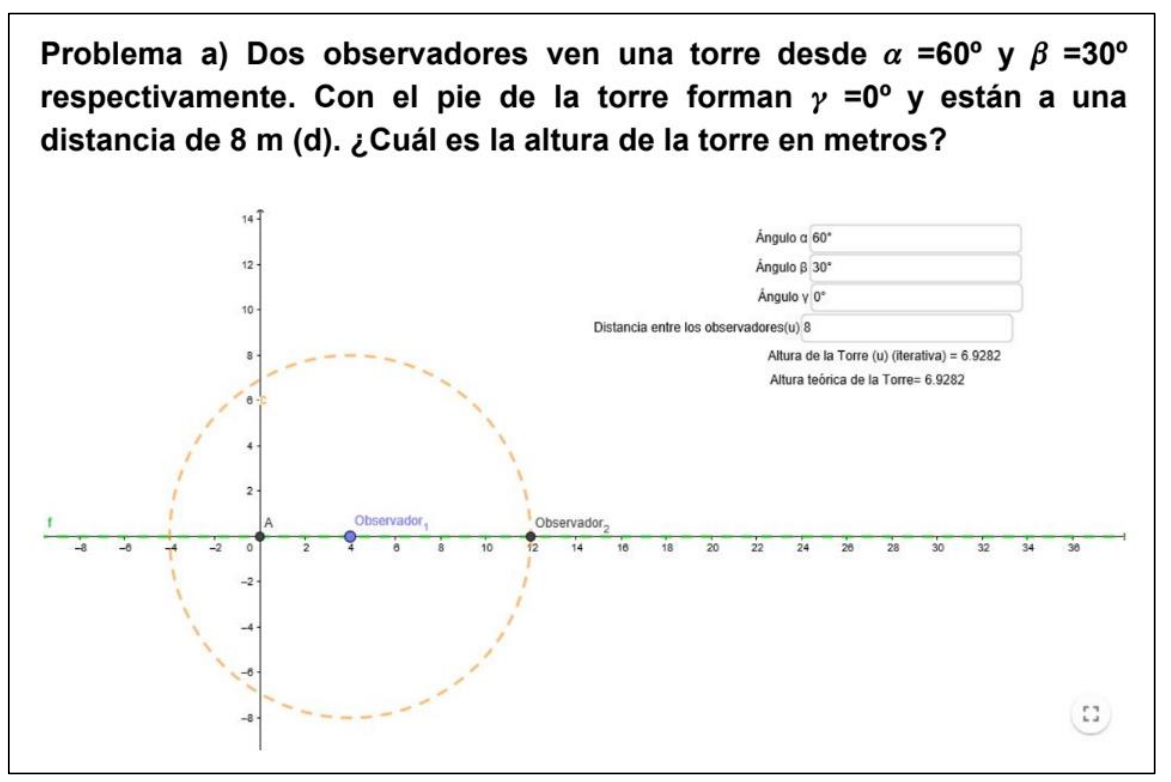

Figura 23. Resolución del problema a) mediante el applet realizado por Carlos.

Como conclusión, Carlos refleja un progreso adecuado de análisis del applet y su aplicación para dar respuesta a las cuestiones matemáticas vinculadas: ha establecido correctamente la relación entre $\mathrm{d}_{1} \mathrm{y} \mathrm{h}$, así como entre $\mathrm{d}_{2} \mathrm{y} \mathrm{d}_{1}$, realizado correctamente los cálculos numéricos propuestos y ha interpretado correctamente el funcionamiento del applet, mejorando considerablemente respecto al cuestionario 1 en que solo fue capaz de realizar correctamente la resolución algebraica del problema.

\section{Caso 2: Marta}

\section{Respuesta de la Tarea 1:}

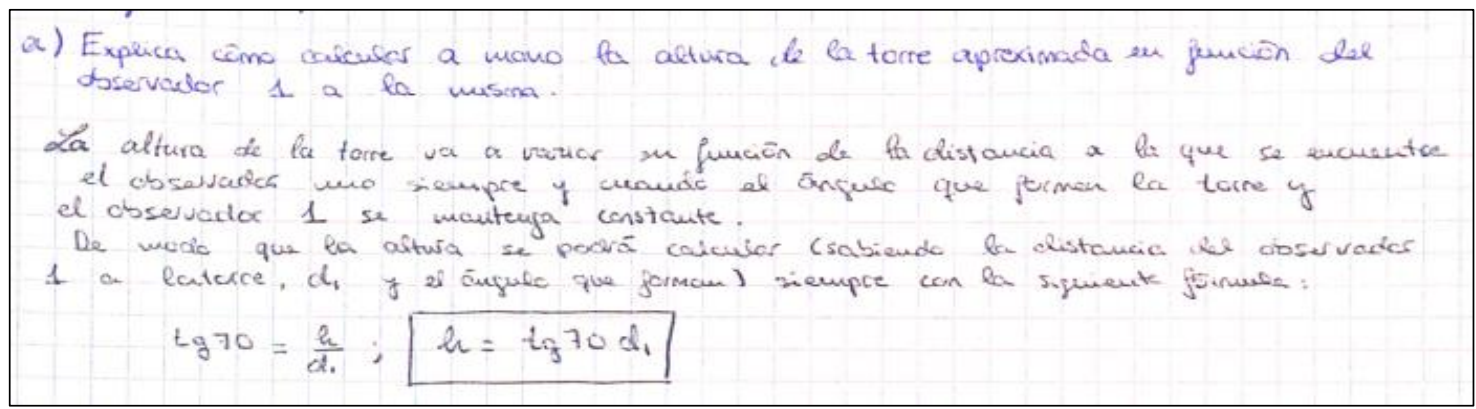

Figura 24. Respuesta de Marta a la Tarea 1 expresando la relación entre la altura de la torre y $\mathrm{d}_{1}$ simbólicamente.

A diferencia de Carlos, Marta hace explícito que el ángulo de visión del observador 1 de la cúspide de la torre se mantendrá constante, por tanto, la altura de la torre variará en función de la distancia del observador 1 a la misma. 


\section{Respuesta del Tarea 2:}

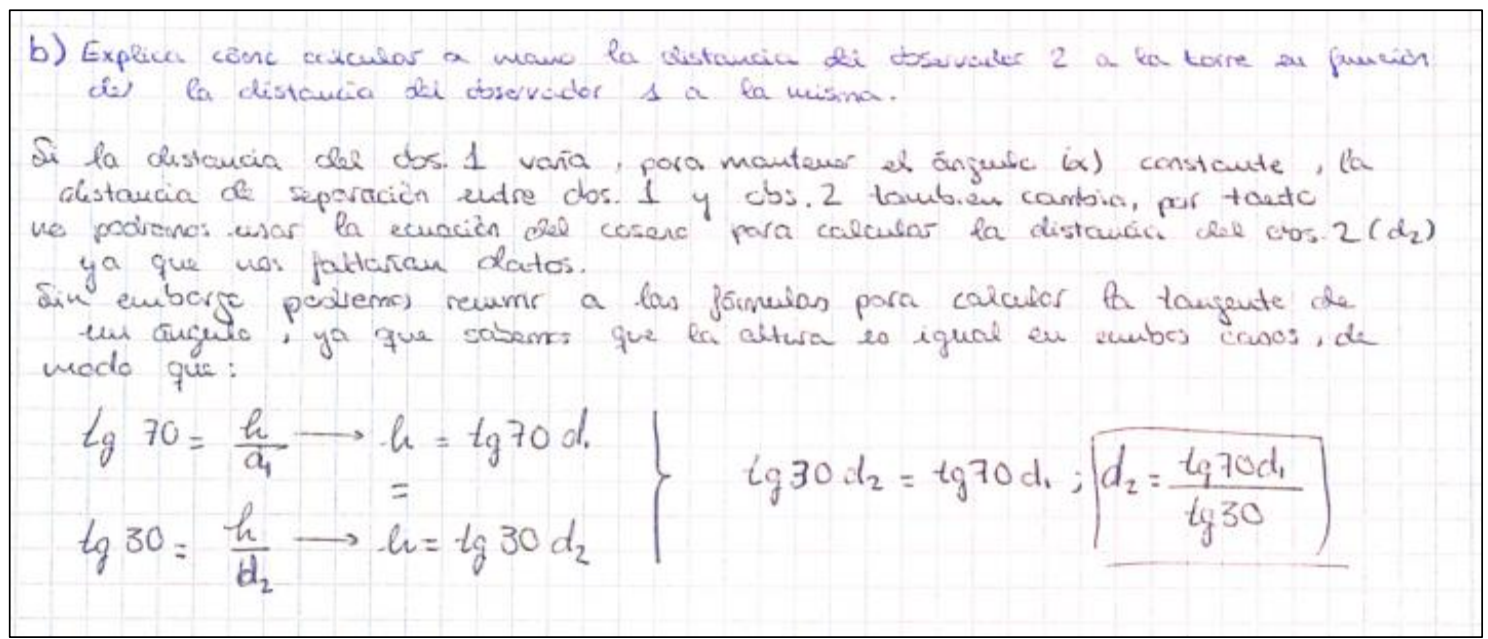

Figura 25. Respuesta de Marta a la Tarea 2 expresando la relación entre $\mathrm{d}_{1} \mathrm{y} \mathrm{d}_{2}$ simbólicamente.

En este caso Marta realiza una aportación muy interesante y es que si el ángulo $\alpha$ se mantiene constante, la distancia entre los observadores va a variar irremediablemente, por eso descarta el Teorema del Coseno. Por otro lado, si se dejara la distancia entre los observadores constante $(8 \mathrm{~m})$, y se usara el Teorema del Coseno para expresar $\mathrm{d}_{2}$ en función de $\mathrm{d}_{1}$, no es posible imponer ángulos de visión fijos porque han variado al dejar la distancia entre los observadores constante. Lo interesante del applet es que reduce todo a un solo parámetro, $\mathrm{d}_{1}$, y la distancia $8 \mathrm{~m}$ impone la solución al buscar el valor óptimo de $\mathrm{d}_{1}$. Esta estudiante además establece la relación esperada y despeja $\mathrm{d}_{2}$.

\section{Respuesta del Tarea 3:}

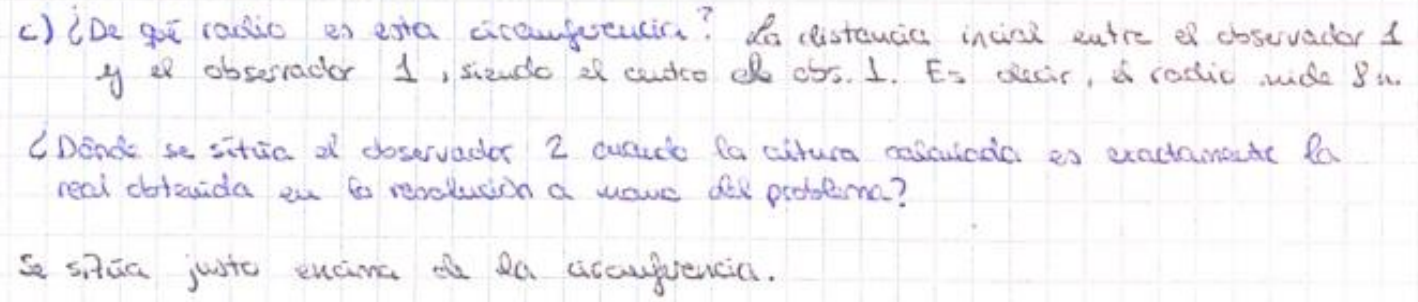

Figura 26. Respuesta de Marta a la Tarea 3 identificando correctamente el radio de la circunferencia y la posición óptima de $\mathrm{O}_{2}$.

En este caso Marta, al igual que Carlos, realiza una identificación adecuada del radio de la circunferencia y de la posición óptima cuando la altura iterativa coincide con la teórica, en la que el observador 2 está sobre la circunferencia de centro observador 1 y radio d. 


\section{Respuesta problemas propuestos:}

Ajusta el primer problema totalmente, el segundo lo ajusta hasta $3.48832 \mathrm{~m}$ frente a los $3.4641 \mathrm{~m}$ teóricos y el tercero lo ajusta hasta $5.78808 \mathrm{~m}$ frente a los $5.77483 \mathrm{~m}$ teóricos.

Como conclusión, Marta muestra un progreso adecuado estableciendo y profundizando en las relaciones matemáticas esperadas y mostrando el uso adecuado del applet. A diferencia de Carlos, hay evidencias de reflexión crítica sobre la elección de los parámetros, y los efectos producidos sobre otras variables involucradas, en este caso, la distancia entre los observadores. Marta también ha mostrado un gran progreso respecto al cuestionario 1, en que solo respondía de forma correcta a la resolución algebraica del problema.

\section{Caso 3: Ana}

\section{Respuesta de la Tarea 1:}

Si cogemos al observador 1 podemos utilizar la tangente de 70 , pero aun así nos haría falta los demás datos para calcular la altura de la torre.

\[ \tan 70=\frac{h}{y} \]
$y=$ distancia del observador $1 \mathrm{a}$ la torre.

Figura 27. Respuesta de Ana a la Tarea 1 considerando que h y d $\mathrm{d}_{1}$ covarían.

Ana deja la relación indicada, pero no expresa la relación explícita entre h y la distancia del observador 1 a la misma, considera en consecuencia que "covarían", es decir, no expresa que la altura dependerá de la distancia del observador 1 a la torre, elegida a conveniencia, sino que deja las dos variables h e y indefinidas. Esto muestra que Ana presenta un obstáculo ya que considera que hacen falta más datos para hallar la altura "real" de la torre, que es cierto, pero no es el objetivo de esta tarea.

\section{Respuesta de la Tarea 2:}

Primero calcularíamos la distancia del observador 1 a la torre con la tangente de 70 y luego con el triángulo que se forma con grado 60 y lado 8 podemos calcular la distancia del observador 2 a la torre. También podemos calcular la distancia del observador 2 a la torre a través de la tangente de 30 pero necesitaríamos primero la altura de la torre. 
En este caso Ana muestra un bloqueo patente, no establece una relación de dependencia entre variables, $\mathrm{d}_{1}$ sigue considerándolo un valor fijo desconocido que depende de la altura de la torre, igualmente no relaciona $\mathrm{d}_{2}$ con $\mathrm{d}_{1}$, solo con la altura de la torre.

\section{Respuesta de la Tarea 3:}

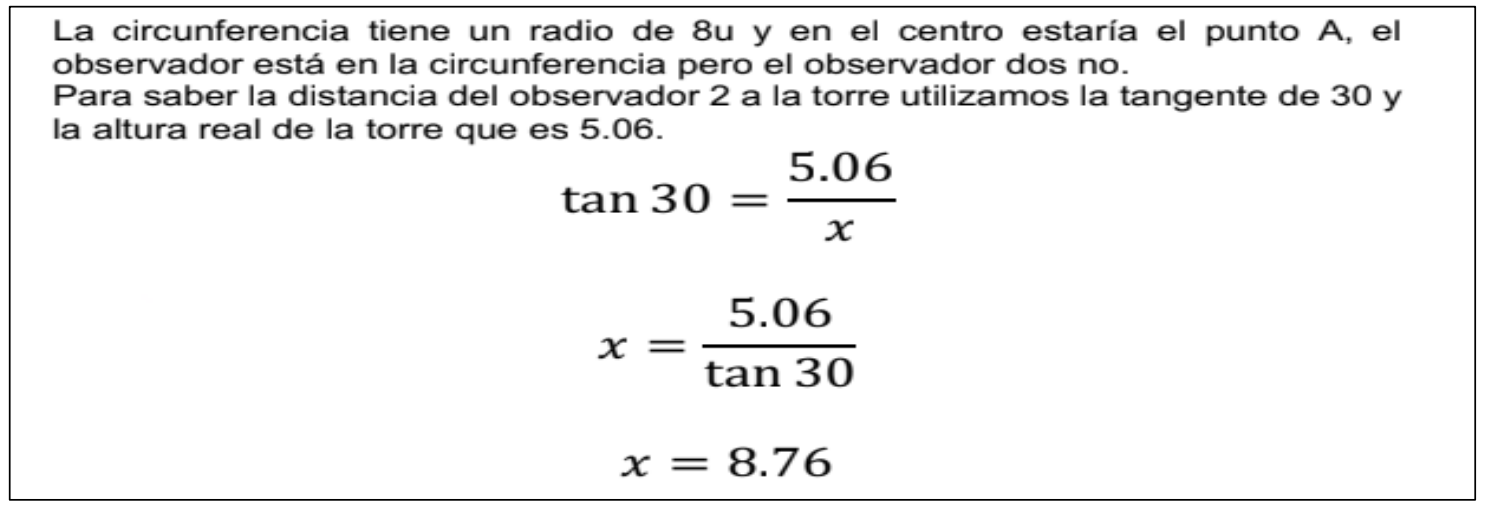

Figura 28. Respuesta de Ana a la Tarea 3. Determina algebraicamente la distancia del observador 2 a la torre en lugar de realizar la interpretación gráfica usando el applet.

Ana recurre a determinar algebraicamente la distancia del observador 2 a la torre en lugar de interpretar gráficamente que este elemento estará sobre la circunferencia de centro observador 1 y radio 8 , dato que identifica adecuadamente. Claramente, se observa que Ana presenta un conflicto cognitivo entre las representaciones gráfica y simbólica, no hay coordinación entre los dos registros de representación.

\section{Respuesta a problemas propuestos:}

Ana ajusta totalmente los dos primeros diagramas, pero en el tercero ajusta hasta 5.78808 $\mathrm{m}$ frente a los $5.77483 \mathrm{~m}$ teóricos.

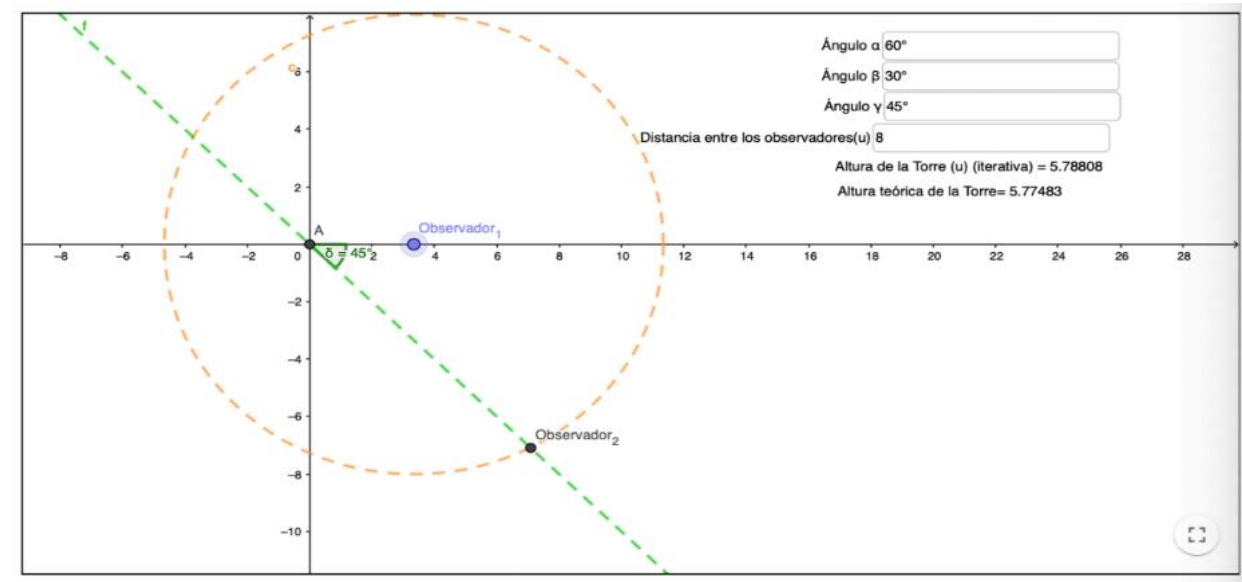

Figura 29. Resolución del problema c) mediante el applet realizado por Ana. 
Como conclusión, Ana parece establecer una ruptura entre el razonamiento algebraico y el gráfico. En particular, no establece una relación de dependencia entre variables, al otorgar el significado de incógnita a las letras fundamentalmente, $\mathrm{y}$, aunque emplea el applet adecuadamente para obtener el resultado esperado, no llega a coordinar adecuadamente los dos registros de representación. Ana ha mostrado cierto progreso respecto al cuestionario 1, pero no es el esperado tras la implementación del cuestionario 2.

\section{Caso 4: Laura}

\section{Respuesta de la Tarea 1:}

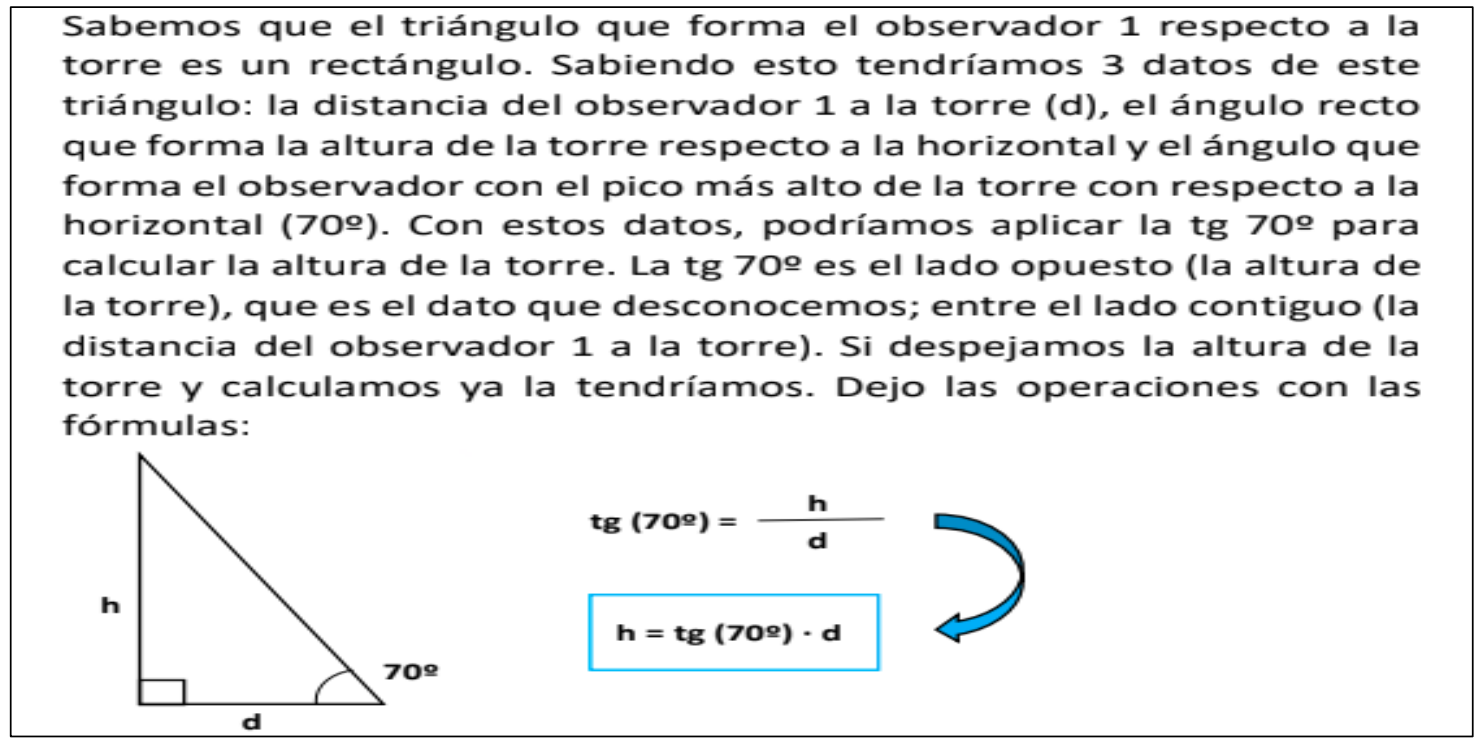

Figura 30. Respuesta de Laura a la Tarea 1 expresando la relación entre la altura de la torre y $\mathrm{d}_{1}$ simbólicamente.

Laura emplea una representación gráfica de la relación Torre-Observador 1, y simbólicamente emplea la relación entre la altura de la torre y la distancia del observador 1 a la misma y despeja h en función de d. 


\section{Respuesta de la Tarea 2:}

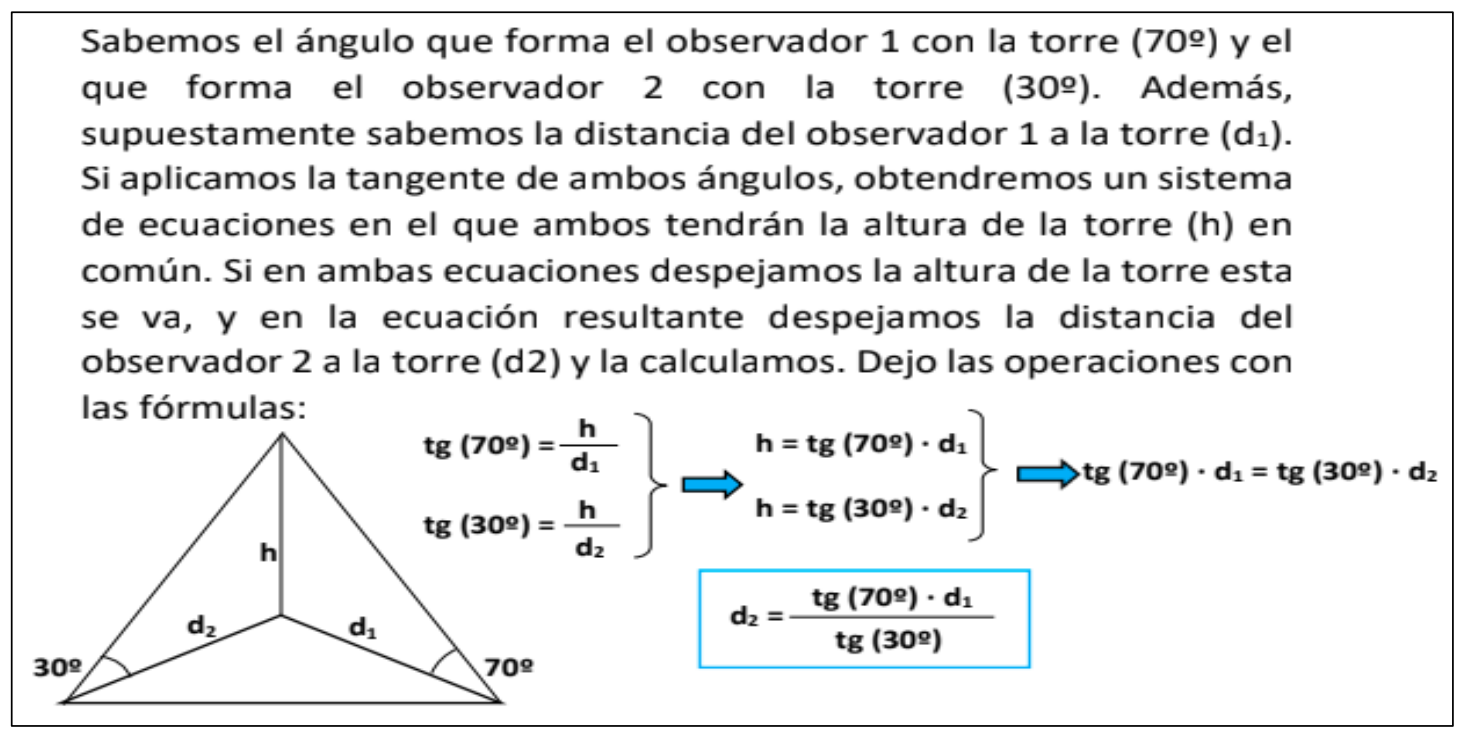

Figura 31. Respuesta de Laura a la Tarea 2 expresando la relación entre $\mathrm{d}_{1} \mathrm{y}^{\mathrm{d}_{2}}$ simbólicamente.

En este caso Laura se apoya en la representación gráfica de la relación Torre-Observador 1-Observador 2, para deducir la relación esperada entre $\mathrm{d}_{1} \mathrm{y} \mathrm{d}_{2}$, despejando finalmente $\mathrm{d}_{2}$ en función de $\mathrm{d}_{1}$.

\section{Respuesta de la Tarea 3:}

\section{El radio de esa circunferencia corresponde con la distancia que separa ambos observadores. \\ Cuando la altura calculada es igual a la real, el observador 2 se encuentra en un punto por el que pasa la circunferencia.}

Figura 32. Respuesta de Laura a la Tarea 3 identificando correctamente el radio de la circunferencia y la posición óptima del observador 2.

Laura interpreta adecuadamente el applet análogamente a Carlos y Marta.

\section{Respuesta problemas propuestos:}

Laura ajusta totalmente los dos primeros diagramas, pero en el tercero ajusta hasta $5.78808 \mathrm{~m}$ frente a los $5.77483 \mathrm{~m}$ teóricos, al igual que Ana.

Como conclusión, Laura muestra un progreso adecuado a lo largo del cuestionario, a diferencia de Carlos y Marta, Laura muestra un apoyo en el esquema gráfico del problema para establecer las relaciones algebraicas correspondientes. Análogamente a todos los 
demás emplea el applet apropiadamente para generar los resultados sobre la altura de la torre en cada uno de los tres problemas alternativos. Laura también ha mostrado un gran progreso respecto al cuestionario 1 , en que solo respondía de forma correcta a la resolución algebraica del problema.

\section{Caso 5: María}

\section{Respuesta de la Tarea 1:}

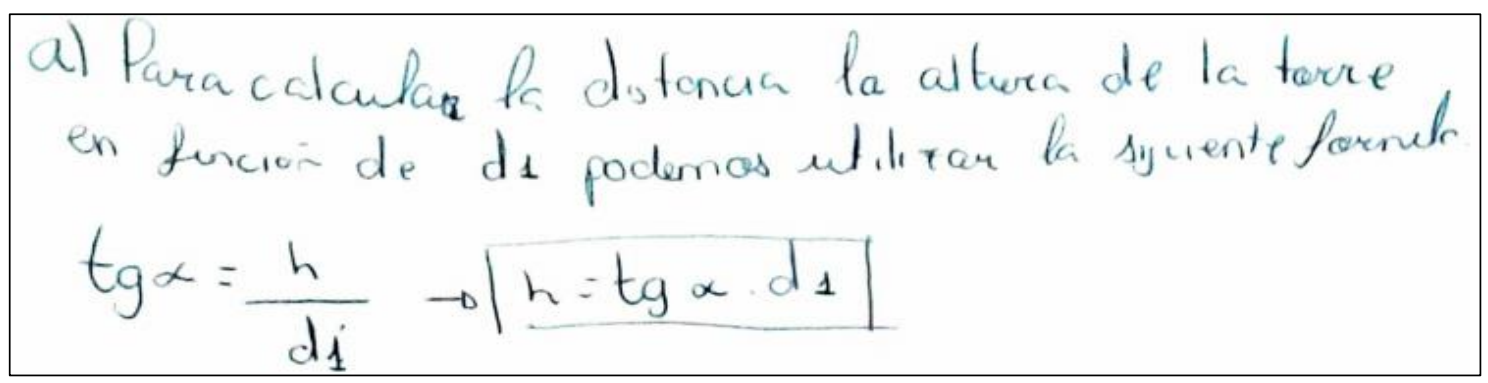

Figura 33. Respuesta de María a la Tarea 1 expresando la relación entre la altura de la torre y d simbólicamente.

En este caso María expresa la relación entre la altura de la torre y $\mathrm{d}_{1}$ empleando exclusivamente el lenguaje simbólico.

\section{Respuesta de la Tarea 2:}

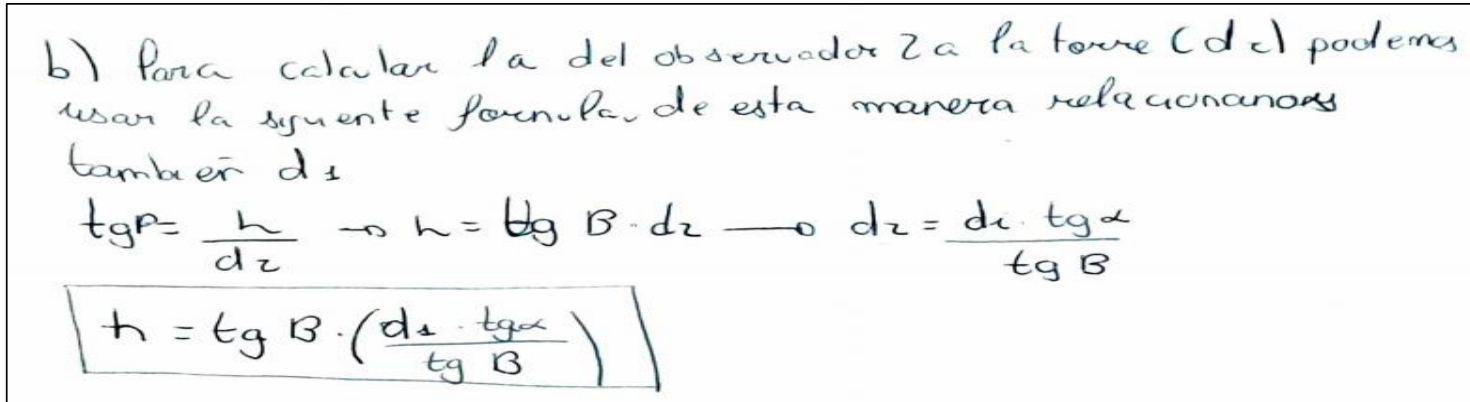

Figura 34. Respuesta de María a la Tarea 2 expresando la relación entre $\mathrm{d}_{1}$ y $\mathrm{d}_{2}$ simbólicamente.

En este caso María establece la relación entre $\mathrm{d}_{1} \mathrm{y} \mathrm{d}_{2}$, pero vuelve otra vez a escribir $\mathrm{h}$ en función de $d_{1}$ originalmente deducida, quizás con la finalidad de comprobar que $\mathrm{d}_{2}$ está bien despejado en función de $\mathrm{d}_{1}$. 


\section{Respuesta de la Tarea 3:}

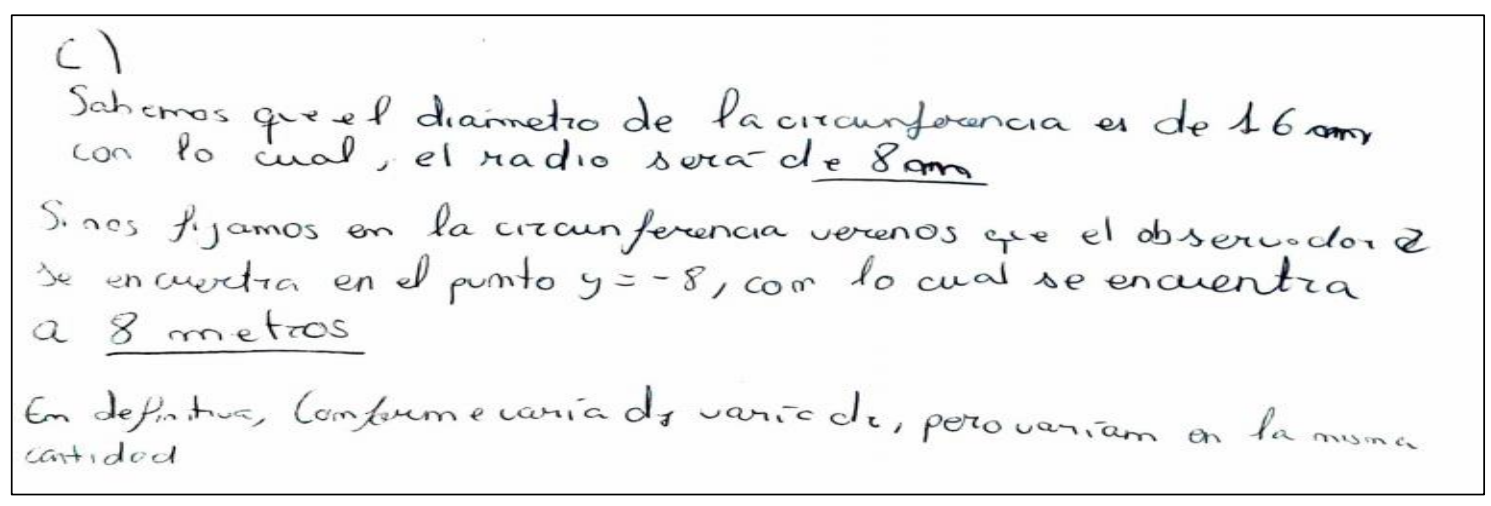

Figura 35. Respuesta de María a la Tarea 3 en la que presenta dificultades para interpretar correctamente el applet.

María presenta un obstáculo ya que al intentar obtener la posición óptima en el applet se fija en la ordenada del punto "Observador 2" que está cerca de y=-8 e informa de que está a $8 \mathrm{~m}$, lo cual no es una inferencia apropiada, porque no siempre se producirá ese hecho, al depender de la situación concreta en la que nos encontremos. El applet distrae la atención del estudiante hacia propiedades que no son generales sino circunstanciales o meras casualidades.

\section{Respuesta problemas propuestos:}

María no ajusta adecuadamente la distancia de $\mathrm{d}_{1}$ para aproximar la altura teórica, en el primer problema alcanza solo $4.23236 \mathrm{~m}$, frente a los $6.9282 \mathrm{~m}$ teóricos. En el segundo problema lo deja en $4.23236 \mathrm{~m}$ frente a los $3.4641 \mathrm{~m}$ teóricos y en el tercer problema, lo deja en $4.23236 \mathrm{~m}$ respecto de los $5.77483 \mathrm{~m}$ teóricos, lo cual informa de que no ha dado utilidad alguna al applet tras la segunda intervención.

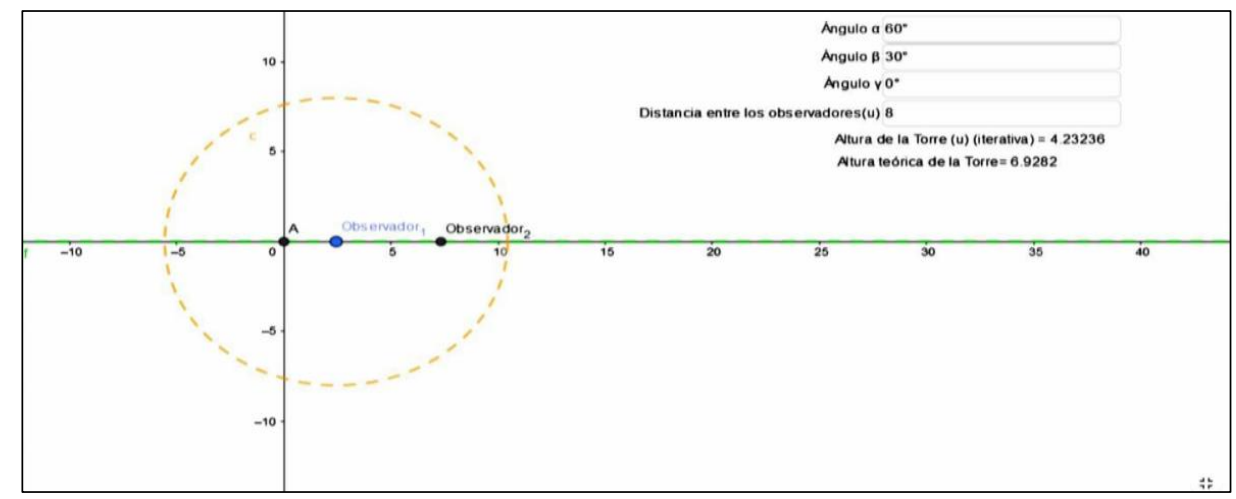

Figura 36. Resolución del problema a) mediante el applet realizado por María. 
Como conclusión, María muestra el perfil más pobre en el dominio de las habilidades algebraicas y gráficas relacionadas con el problema. En relación con el dominio algebraico llega a establecer las relaciones entre $\mathrm{h}$ y $\mathrm{d}_{2}$ con $\mathrm{d}_{1}$, sin embargo, en relación con el dominio gráfico, no llega a establecer la necesidad de que el observador 2 esté en la circunferencia de centro observador 1 y radio $8 \mathrm{~m}$, estableciendo inferencias inapropiadas. María ha mostrado un progreso respecto al cuestionario 1, pero no es el esperado tras las indicaciones dadas en el cuestionario 2.

\section{Análisis conjunto de la Tarea 4}

Para el caso de la Tarea 4, vamos a realizar un análisis conjunto de las respuestas ya que de esta manera se puede observar las semejanzas y diferencias respecto a los resultados numéricos obtenidos por los cinco estudiantes.

Tarea 4: "Completa las tablas siguientes y calcula la distancia del observador 2 a la torre y la altura de la torre a partir de diferentes distancias del observador 1 a la misma para el caso inicial, es decir, el caso en el que dos observadores ven una torre desde $70^{\circ}$ y $30^{\circ}$ respectivamente. Con el pie de la torre forman $60^{\circ}$ y están a una distancia de $8 \mathrm{~m}(\mathrm{~d}) . "$

Los resultados numéricos aportados para esta tarea por los 5 estudiantes se muestran en la Tablas 1 y 2 siguientes:

Tabla 1. Respuestas de los 5 alumnos al cálculo de la distancia $\mathrm{d}_{2}$ a partir de diferentes distancias $\mathrm{d}_{1}$ dadas.

\begin{tabular}{|c|c|c|c|c|c|}
\hline $\begin{array}{c}\mathrm{d}_{1} \text { (Distancia } \\
\text { del observador } \\
1 \text { a la Torre) }\end{array}$ & $\mathrm{d}_{2}$ (Carlos) & $\mathrm{d}_{2}$ (Marta) & $\mathrm{d}_{2}$ (Ana) & $\mathrm{d}_{2}$ (Laura) & $\mathrm{d}_{2}$ (María) \\
\hline $2 \mathrm{~m}$ & $9.52 \mathrm{~m}$ & $9.5175 \mathrm{~m}$ & $\begin{array}{c}\text { Usa Teorema } \\
\text { del Coseno } \\
\text { imponiendo } \\
\mathrm{d}=8 \mathrm{~m}\end{array}$ & $9.52 \mathrm{~m}$ & $9.64 \mathrm{~m}$ \\
\hline $4 \mathrm{~m}$ & $19.04 \mathrm{~m}$ & $19.0350 \mathrm{~m}$ & $\begin{array}{c}\text { Usa Teorema } \\
\text { del Coseno } \\
\text { imponiendo } \\
\mathrm{d}=8 \mathrm{~m}\end{array}$ & $19.04 \mathrm{~m}$ & $19.28 \mathrm{~m}$ \\
\hline $8 \mathrm{~m}$ & $38.08 \mathrm{~m}$ & $38.07016 \mathrm{~m}$ & $\begin{array}{c}\text { Usa Teorema } \\
\text { del Coseno } \\
\text { imponiendo } \\
\mathrm{d}=8 \mathrm{~m}\end{array}$ & $38.08 \mathrm{~m}$ & $38.5 \mathrm{~m}$ \\
\hline
\end{tabular}


Carlos, Marta y Laura rellenan adecuadamente la tabla, Ana emplea el Teorema del Coseno, y María da valores aproximados que difieren de los de sus compañeros al haber aplicado la aproximación solo hasta las décimas y posteriormente el redondeo. Los cálculos que realiza Ana mediante el Teorema del Coseno carecen de sentido en este caso ya que imponiendo la distancia $\mathrm{d}=8 \mathrm{~m}$, si la distancia del observador 1 a la torre cambia, los ángulos $\beta$ y $\gamma$ varían necesariamente.

Tabla 2. Respuestas de los 5 alumnos al cálculo de la altura de la torre a partir de diferentes distancias $\mathrm{d}_{1}$ dadas.

\begin{tabular}{|c|c|c|c|c|c|}
\hline $\begin{array}{c}\mathrm{d}_{1} \text { (Distancia } \\
\text { del observador } \\
1 \text { a la Torre }\end{array}$ & $\mathrm{h}$ (Carlos) & $\mathrm{h}$ (Marta) & $\mathrm{h}$ (Ana) & $\mathrm{h}$ (Laura) & $\mathrm{h}$ (María) \\
\hline $2 \mathrm{~m}$ & $5.49 \mathrm{~m}$ & $5.494905 \mathrm{~m}$ & $5.49 \mathrm{~m}$ & $5.49 \mathrm{~m}$ & $5.49 \mathrm{~m}$ \\
\hline $4 \mathrm{~m}$ & $10.98 \mathrm{~m}$ & $10.79079 \mathrm{~m}$ & $10.99 \mathrm{~m}$ & $11 \mathrm{~m}$ & $10.98 \mathrm{~m}$ \\
\hline $8 \mathrm{~m}$ & $21.97 \mathrm{~m}$ & $21.97982 \mathrm{~m}$ & $21.98 \mathrm{~m}$ & $21.98 \mathrm{~m}$ & $21.97 \mathrm{~m}$ \\
\hline
\end{tabular}

En este caso todos afinan la altura aproximada de la torre empleando diferentes estrategias de truncamiento y redondeo. 


\section{CAPÍTULO 5}

\section{CONCLUSIONES}

En este capítulo se va a sintetizar las conclusiones tras el proceso de investigación. Para ello analizaremos el grado de cumplimiento de los objetivos, su relación con la literatura, algunas limitaciones presentadas y finalizaremos mencionando algunas líneas de investigación abiertas a raíz del trabajo realizado.

El objetivo propuesto fue el siguiente:

"Explorar y describir las nociones, representaciones y conceptos que los estudiantes ponen de manifiesto cuando resuelven un problema trigonométrico de forma escrita y mediante el uso de las Tecnologías de la Información y la Comunicación.”

Se han cubierto las expectativas ya que la información recogida ha sido muy útil para realizar un análisis amplio y establecer relaciones entre las nociones y los conceptos que los estudiantes emplean en la resolución simbólica y gráfica del problema trigonométrico propuesto.

El objetivo general del estudio lo desglosamos según cuatro objetivos específicos, cuyos logros pasamos a describir.

\subsection{Logro del primer objetivo específico.}

El primer objetivo específico fue el siguiente:

1. Diseñar e implementar un instrumento con el cual recoger información en registro verbal, simbólico y gráfico referente al problema trigonométrico propuesto.

Para lograr este primer objetivo se llevó a cabo una revisión de libros de texto mediante la cual se recogió información útil para el diseño y construcción del instrumento con el cual llevamos a cabo el estudio.

En el primer capítulo de este trabajo se ha delimitado el problema y se ha justificado la importancia de la realización del mismo.

Además, en el segundo capítulo se han expuesto los fundamentos teóricos del estudio que han ayudado a fundamentar correctamente el instrumento. Así, en el apartado 2.1 se ha presentado la noción de sistema de representación, que hemos sometido a revisión crítica mediante contraste con otras interpretaciones. En el apartado 2.2 se ha introducido la 
noción de significado de un concepto matemático escolar que es clave en este estudio para la realización del análisis conceptual y del análisis del contenido realizado posteriormente. En el apartado 2.3. se ha introducido la noción de génesis instrumental que permite explicar la evolución del alumnado durante la realización de los cuestionarios, volviendo a invertir esquemas de utilización familiares ya constituidos o produciendo nuevos esquemas de uso que le permiten alcanzar los objetivos contemplados.

Por último, en el apartado 2.4. se ha presentado un balance de la revisión de investigaciones sobre la enseñanza de la trigonometría. Se han abordado investigaciones referentes a las técnicas para la enseñanza de la trigonometría, el uso de las nuevas tecnologías para el aprendizaje de la trigonometría, el estudio de las dificultades y errores en el aprendizaje de la trigonometría y los significados de las razones trigonométricas que manifiestan los estudiantes. Estos documentos han ayudado a delimitar el estudio, proporcionando focos de interés para su abordaje y aportando antecedentes.

Una vez redactado el primer cuestionario, se procedió a la aplicación del mismo llevando a cabo la fase empírica del trabajo de investigación. Tras la observación de un uso inesperado del applet por parte del alumnado se determinó la realización de un segundo cuestionario con instrucciones de forma más pautada con la finalidad de profundizar en la interpretación gráfica del problema haciendo uso del applet.

\subsection{Logro del segundo objetivo específico.}

El segundo objetivo específico fue el siguiente:

2. Describir el método de resolución llevado a cabo por los estudiantes y establecer sus características principales.

Para el logro de este objetivo se procedió a la recopilación de todas las producciones de los estudiantes de la Tarea 1 del cuestionario 1 y a un análisis cualitativo de las mismas mediante un análisis conceptual y de contenido de las producciones del alumnado.

A continuación, realizamos un resumen de las categorías extraídas en el análisis de la Tarea 1 del cuestionario 1 .

Los temas principales que se encuentran en las respuestas del alumnado para esta tarea lo forman el uso de esquema y el lenguaje algebraico. 
Respecto al tema "uso de esquema" se encontraron 5 unidades de análisis: los elementos no relevantes del problema, los triángulos representados, los ángulos representados, las incógnitas marcadas sobre los lados de los diferentes triángulos y la distancia entre los dos observadores.

Para la unidad de análisis de elementos no relevantes, observamos la presencia de monigotes o figuras que simulaban a los observadores y que no son características estructurales del problema, así como la presencia de ángulos no dados en el problema y que no son necesarios para resolver el problema.

Para la unidad de análisis triángulo, observamos que había alumnos que identificaban la presencia de triángulos rectángulos y lo señalizaban con el símbolo del ángulo recto y otros que no los identificaban.

Para la unidad de análisis ángulo, observamos que había alumnos que marcaban los ángulos interiores agudos con un arco indicando así su amplitud y el ángulo interior recto con el símbolo del ángulo recto.

Para la unidad de análisis incógnitas marcadas sobre los lados de los diferentes triángulos, había alumnos que marcaron las incógnitas del problema ( $\mathrm{a}, \mathrm{b}$ y h) y otros que además marcaban, de forma innecesaria, como incógnitas (X e Y) las hipotenusas de los triángulos rectángulos.

Para la unidad de análisis distancia entre los dos observadores, había alumnos que indicaban directamente la distancia entre los observadores y otros que la indicaban de forma implícita mediante la letra $d$.

Sobre el tema "lenguaje algebraico" no existen diferencias en el alumnado ya que éstos emplean un método de resolución estandarizado y cuadriculado.

\subsection{Logro del tercer objetivo específico.}

El tercer objetivo específico fue el siguiente:

3. Establecer relaciones entre el uso de la representación simbólica y la representación gráfica dada por el applet en la resolución del problema trigonométrico propuesto.

Para el logro de este objetivo se procedió a la recopilación de todas las producciones de los estudiantes de las Tareas 2, 3, 4 y 5 del cuestionario 1 y a un análisis cualitativo de 
las mismas mediante un análisis conceptual y de contenido de las producciones del alumnado para establecer relaciones entre el uso de la representación simbólica y la representación gráfica dada por el applet.

A continuación, realizamos un resumen de las categorías extraídas en el análisis.

En la Tarea 2 del cuestionario 1 se identificaron 3 categorías de respuesta: los que enfatizaban en la resolución algebraica del problema sin obtener la potencialidad del applet como estimador de la solución, los que buscaban alternativas a la finalidad del applet sin abordar la auténtica finalidad del mismo y los que simplemente destacaban aspectos superficiales del applet y no las relaciones matemáticas que se establecen en él.

En la Tarea 3 del cuestionario 1 no se han establecido categorías puesto que el 100\% de los estudiantes parte de la distancia del observador 1 solución para calcular la altura de la torre teórica.

En la Tarea 4 del cuestionario 1 se identificaron 3 categorías de respuesta: los que establecían relaciones entre variables, pero no identifican explícitamente el papel del applet en establecerlas; los que consideran que sin datos concretos no se puede calcular $\mathrm{d}_{2}$ porque hace falta resolver una ecuación y los que parten de los resultados solución para establecer relaciones.

En la Tarea 5 del cuestionario 1 no se establecieron categorías puesto que el 100\% de los estudiantes empleaba la razón trigonométrica tangente para hallar la distancia del $\mathrm{O}_{1}$ a partir de la altura teórica de la torre.

Como conclusión, pudimos apreciar que ningún estudiante fue capaz de establecer una relación entre la representación simbólica y la representación gráfica mediante el applet del problema propuesto. Todos resolvieron correctamente la Tarea 1 que implicaba la resolución algebraica del problema, pero no resolvieron correctamente las tareas y problemas que involucraban el uso del applet.

\subsection{Logro del cuarto objetivo específico.}

El cuarto objetivo específico fue el siguiente:

4. Describir el progreso realizado por los estudiantes en la resolución del problema trigonométrico planteado y en el empleo del applet relacionado. 
Para el logro de este objetivo se ha partido del análisis de casos del cuestionario 2 que ha permitido determinar la evolución de los estudiantes de forma transversal a lo largo del mismo y en relación con el cuestionario 1 y establecer semejanzas y diferencias con las respuestas de sus compañeros.

Tras la implementación y el análisis del segundo cuestionario pudimos apreciar un claro progreso en el alumnado: Carlos, Marta y Laura fueron capaces de establecer relaciones entre la representación gráfica y simbólica, sin embargo, Ana y María, aunque mostraron progresos respecto al primer cuestionario, manifiestan bloqueos en el dominio algebraico (Atribución exclusiva de significado de incógnitas a las letras) y gráfico (No realiza correctamente la resolución gráfica del problema), respectivamente.

\subsection{Relación con la literatura.}

Como resultado emergente de esta investigación mostramos el trabajo de Puig (2012) que hace referencia a los diferentes significados de las letras. Puig (2012) afirma que el álgebra en el currículo de secundaria ha de presentarse, al menos, desde tres puntos de vista: el álgebra como un sistema de signos en que realizar los procesos de generalización, abstracción y demostración; el álgebra como un instrumento para la resolución de problemas a través de la traducción de éstos a sistemas de ecuaciones o gráficas de funciones, y el álgebra como sistema de signos que permite que los fenómenos modelados mediante funciones se organicen en familias, cuyas características se establecen y se estudian en el plano de la expresión.

El segundo de los aspectos corresponde a la resolución algebraica de problemas de enunciado verbal, es decir, a la resolución de problemas según el Método Cartesiano. En este caso, las letras tienen significado de incógnita.

El tercero de los aspectos tiene relación con los procesos de modelización. En este caso, las expresiones algebraicas representan relaciones funcionales y su significado está ligado a los procesos de traducción entre ellas, las tablas de datos numéricos y las representaciones gráficas cartesianas con un software que combine un sistema de cálculo simbólico con una pantalla gráfica y una hoja de cálculo, como GeoGebra. En este caso, las letras tienen significado de variable.

El cuestionario realizado por el alumnado tenía tareas pertenecientes al segundo aspecto (resolución algebraica del problema) y al tercer aspecto (modelado y resolución del 
problema mediante el uso del applet), por lo que dependiendo de la tarea las letras tenían significado de incógnita o de variable.

Tras el análisis de las respuestas del cuestionario 1 realizado por el alumnado hemos podido comprobar que todos los estudiantes consideraron en un primer término, tras la manipulación del applet, que la distancia del observador 1 era un valor desconocido, una incógnita, en lugar de una variable que podía tomar valores libremente. Ello pudo deberse a que el alumnado no interpretó correctamente el funcionamiento del applet y, en consecuencia, no interpretó correctamente el significado asociado a $\mathrm{d}_{1}$.

Además, como consecuencia de las dificultades respecto a la interpretación gráfica del problema se ha producido en el alumnado un conflicto cognitivo entre la representación gráfica y la representación simbólica del problema. Sin embargo, estas dificultades fueron resueltas por gran parte del alumnado tras la implementación de un nuevo cuestionario en el que se les daba unas nuevas pautas para facilitar la interpretación gráfica del problema. El alumnado ha ido evolucionando durante el curso de las tareas, volviendo a invertir esquemas de utilización familiares ya constituidos o produciendo nuevos esquemas de uso que les ha permitido alcanzar los objetivos contemplados, llevando a cabo el proceso denominado como Génesis Instrumental que forma parte del Enfoque Instrumental de Rabardel (1995).

Como ya comentamos en los antecedentes de este trabajo, existen muy pocas investigaciones en las que se ponga en práctica el aprendizaje y la enseñanza de la trigonometría mediante el uso de las nuevas tecnologías (Zengin, Furkan y Kutluca, 2011; Prieto, 2015; Posada-Acosta; 2017). Los resultados de esta investigación han sido muy enriquecedores ya que han permitido corroborar lo que otros investigadores ya afirmaron y es que una instrucción asistida con un software, como suplemento para promover un aprendizaje constructivista, es más efectivo que una metodología tradicional.

\subsection{Limitaciones de la investigación.}

La muestra seleccionada en este estudio ha sido seleccionada de forma intencionada. Los participantes han sido elegidos en función de la disponibilidad del centro y de los docentes, y contando con la aceptación del alumnado. Los sujetos cursan primer curso de Bachillerato un centro de Educación Secundaria Obligatoria de la provincia de Huelva. Debido a la elección no aleatoria de los mismos, la validez externa acota el alcance de nuestros hallazgos a dicho centro docente. 
Además, debido a la situación sanitaria actual, no fue posible la realización de un taller previo para abordar el problema inicial y establecer unas pautas para la realización del cuestionario inicial. Ello dificultó la comprensión del funcionamiento del applet por parte de los estudiantes y por ello fue necesario la realización de un segundo cuestionario incluyendo nuevas pautas.

Por otro lado, el estudio es exploratorio y tiene interés por la escasez de investigaciones sobre este tema. No se pretende generalizar los resultados sino obtener evidencias sobre modos de resolver un problema trigonométrico de forma simbólica y gráfica en un contexto particular.

\subsection{Sugerencias para investigaciones futuras.}

Las limitaciones enumeradas nos orientan hacia nuevas líneas de investigación. Así, convendría realizar esta misma investigación con un mayor número de sujetos para que los resultados fueran significativos.

Además, sería necesario para una correcta interpretación y resolución de los problemas propuestos, la realización de un taller previo en el que el alumnado pueda expresar y aclarar sus dudas previamente a la realización de los cuestionarios ya que la resolución de problemas geométricos entraña gran dificultad para el alumnado. A menudo, los contenidos geométricos son abordados a finales de curso dando prioridad a otros contenidos tales como los bloques de números y álgebra, funciones o estadística, provocando en el alumnado grandes conflictos para la interpretación y resolución de problemas geométricos.

Los resultados extraídos han sido relevantes puesto que no existe muchas investigaciones que aborden la razón trigonométrica tangente, y en particular, la resolución de problemas trigonométricos mediante el uso de un applet. Por ello, sería interesante seguir trabajando en este campo, incluyendo nuevos problemas trigonométricos que sean abordables mediante el uso de las nuevas tecnologías. Ello sería de gran utilidad en el alumnado ya que tal y como hemos visto en los resultados extraídos, los estudiantes manejan con gran soltura la resolución algebraica de los problemas, pero no la resolución gráfica. 


\section{REFERENCIAS}

Arenas, F., Becerra, M., Morales, F., Urrutia, L. y Gómez, P. (2012). Razones trigonométricas. En P. Gómez (Ed.), Diseño, implementación y evaluación de unidades didácticas matemáticas en MAD 1 (pp. 342-414). Bogotá: Universidad de los Andes.

Army, P. D. (1991). An approach to teaching college course in trigonometry using applications and a graphing calculator. Unpublished doctoral dissertation, Rutgers University, New Brunswick, NJ.

Berthelot, R. y Salim, M. H. (1994). L'enseignement de la géométrie à l'école primaire. [La enseñanza de la geometría en la escuela primaria]. Grand N, 53, 33-56.

Blackett, N. y Tall, D. (1991). Gender and the versatile learning of trigonometry using computer software. En F. Furinghetti (Ed.), Proceedings of the 15th conference of the International Group for the Psychology of Mathematics Education (Vol.1, pp. 144-151). Assisi, Italy: PME.

Bressoud, D. (2010). Historical reflections on teaching trigonometry. Mathematics Teacher, 104 (2), 106-112.

Brown, S. A. (2005). The trigonometric connections: Students' understanding of sine and cosine. Unpublished doctoral dissertation, Illinois State University, Illinois.

Castro, E. y Castro, E. (1997). Representaciones y modelización. En L. Rico (Ed.), La educación matemática en la enseñanza secundaria (pp. 95-124). Barcelona, España: Horsori.

Coleman, H., y Unrau, Y. A. (2005). Social work: Research and evaluation. Quantitative and qualitative approaches. (R. M. Grinnell y Y. A. Unrau, Eds.) (7 ed.). Nueva York, NY: Oxford University Press.

Díaz, S. y Prieto, J. (2015). Sobre los signos de las razones trigonométricas con Geogebra, una manera de trascender las reglas nemotécnicas. En R. Flores (Ed.), Acta Latinoamericana de Matemática Educativa (pp. 1647-1655). México, DF: Comité Latinoamericano de Matemática Educativa.

Domínguez Alfonso, R. D. (2011). Reconsiderando el papel de los docentes ante la sociedad de la información.Etic@ net 11, 179-195.

Escudero, A. M. y Domínguez, J. (2014). De los errores identificados en la investigación a los errores encontrados en un aula de primero de bachillerato. Revista didáctica de las matemáticas, 86, 111-130. 
Frege, G. (1998). Sobre sentido y referencia. En L. M. Valdés (Ed.), Ensayos de Semántica y Lógica (pp. 84-111). Madrid: Tecnos.

Guerrero, Y. A. y Vega, N. P. (2016). Estudio de dificultades y errores en la resolución de triángulos utilizando teorema del seno y el coseno. Comunicación presentada en Encuentro Distrital de Educación Matemática (8-10 de Septiembre). Bogotá, Colombia. Recuperado de: http://funes.uniandes.edu.co/9992/

Héctor Ponce (2000). Enseñar y Aprender Matemática. México: Ediciones Novedades Educativas.

Itzcovich, H. (2005). Iniciación al Estudio Didáctico de la Geometría. De las construcciones a las demostraciones. Buenos Aires, Argentina: Libros del Zorzal.

Kamber, D. y Takaci, D. (2018). On problematic aspects in learning trigonometry. International Journal of Mathematical Education in Science and Technology, 49 (2), 161-175.

Kendal, M., y Stacey, K. (1997). Teaching trigonometry. Vinculum, 34(1), 1-8.

Mansfield, R. M. (1972). Trigonometry with applications. Belmont, MS: Wadsworth.

Markel, D. (1982). Trigonometry -Forgotten and Abused? School Science and Mathematics, 82(7), 548-551.

Martín-Fernández, E., Ruiz Hidalgo, J. F. y Rico, L., (2016) Significado escolar de las razones trigonométricas elementales. Enseñanza de las Ciencias, 34(3), 51-71.

Martín-Fernández, E., Ruiz-Hidalgo, J. F., \& Rico, L. (2019). Meaning and Understanding of School Mathematical Concepts by Secondary Students: The Study of Sine and Cosine. Eurasia Journal of Mathematics, Science and Technology Education, 15(12), 1-18.

Mateo, J. A., Sánchez, F., y Valera, F. J., (1996). La cartografía como experiencia didáctica en educación secundaria obligatoria. Murcia: Universidad de Murcia.

Mathematical Association of England. (1950). The teaching of trigonometry in schools: A report prepared for the Mathematical Association. London: George Bell y Sons, Ltd.

McMillan, J. H., y Schumacher, S. (2005). Investigación Educativa (5ª edición). Madrid: Pearson Addison Wesley.

OCDE (2017), Marco de Evaluación y de Análisis de PISA para el Desarrollo: Lectura, matemáticas y ciencias, Versión preliminar, OECD Publishing, Paris. 
Plazas Rojas, L. J. (2012). Enseñanza de elementos básicos de trigonometría en la astronomía: una propuesta para trabajar con estudiantes de educación media. Trabajo fin de Máster. Universidad Nacional de Colombia, Bogotá.

Posada-Acosta, M., Matilla-Arias, J., y Rosales-Sáez, F. (2017). Potencialidades del software geogebra en la enseñanza de la matemática: estudio de caso de su aplicación en la trigonometría. Roca. Revista Científico - Educacional De La Provincia Granma, 13(4), 401-415.

Puig, L. (2012). Observaciones acerca del propósito del álgebra educativa. En A. Estepa, Á. Contreras, J. Deulofeu, M. C. Penalva, F. J. García y L. Ordóñez (Eds.), Investigación en Educación Matemática XVI (pp. 3 - 20). Jaén: SEIEM

Rabardel, P. (1995). Les hommes et les technologies. Une approche cognitive des instruments contemporains. París: Armand Collins.

Rico, L. (2009). Sobre las nociones de representación y comprensión en la investigación en educación matemática. PNA, 4 (1), 1-14.

Rico, L. (2012). Aproximación a la investigación en Didáctica de la Matemática. Avances de Investigación en Educación Matemática, 1, 39-63.

Rico, L. (2013). El método del Análisis Didáctico. Revista iberoamericana de educación matemática. Unión, 33, 11-27.

Rodríguez Arós, A., Blanco, F. y Muiños, M. J. (2012). Trigonometría plana y esférica con aplicaciones a la navegación. Madrid: Paraninfo.

Sampieri, R., Fernández, C., y Baptista, M. P. (2010). Metodología de investigación (5ª edición). Mexico: McGraw-Hill.

Thompson, K. (2007). Student's understanding of trigonometry enhanced through the use of a real world problem: improving the instructional sequence. Unpublished doctoral dissertation, Illinois State University, Illinois, IL.

Trouche, L. (2004). Managing the complexity of human/machine interactions in computerized learning environments: guiding students' command process through instrumental orchestrations. International Journal of Computers for Mathematical Learning, 9(3), 281-307.

Vizmanos, J. R., y Hernández, J. (2008). Matemáticas $1^{\circ}$ Bachillerato. Ciencias y Tecnología. Madrid: SM.

Weber, K. (2005). Student's understanding of trigonometric functions. Mathematics Education Research Journal, 102(2), 144-147. 
Zengin, Y., Furkan, H. y Kutluka, T. (2011). The effect of dynamic mathematics software Geogebra on student achievement in teaching of trigonometry. Procedia-Social and Behavioral Sciencies, 31, 183-187. 
ANEXOS

\section{Anexo I-Cuestionario 1}

Dos observadores ven una torre desde $\alpha=70^{\circ}$ y $\beta=30^{\circ}$ respectivamente. Con el pie de la torre forman $\gamma=60^{\circ}$ y están a una distancia de $8 \mathrm{~m}(\mathrm{~d})$. ¿Cuál es la altura de la torre en metros? La siguiente ilustración muestra un esquema de la situación descrita en el problema.

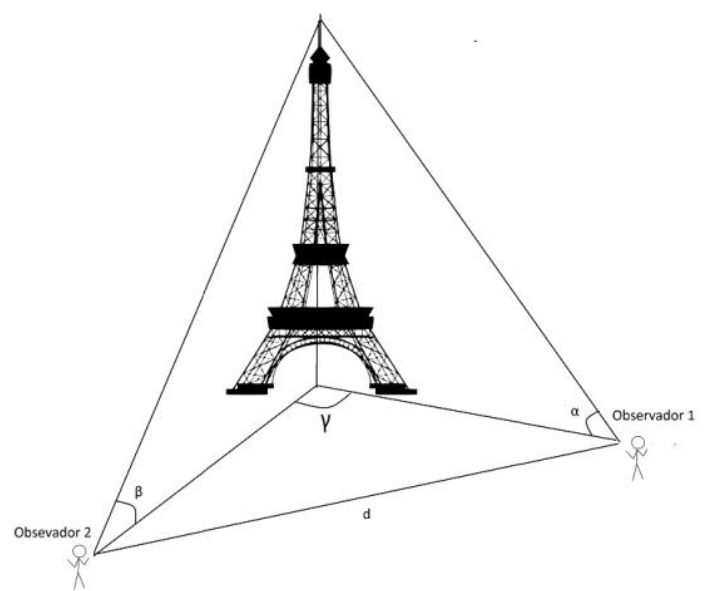

Para ayudarte a resolver este problema se ha diseñado un applet con Geogebra. Puedes verlo en el siguiente enlace: https://www.geogebra.org/m/bwretzcp

1. Resuelve en primer el lugar el problema con ayuda de la calculadora, expresando los cálculos convenientes a mano. Con la intención de que puedas comprobar si tus cálculos han sido apropiados, el applet te facilita la solución 5,06 m, pero nos interesa la forma en la que has llegado a esa solución.

Tras observar el applet responde a las siguientes preguntas:

2. ¿Podrías explicar tras manipularlo en qué se basa? Explica cómo funciona.

3. ¿Cómo se ha calculado el valor "aproximado" de la altura de la torre a partir de la posición del observador 1? Expresa los cálculos necesarios.

4. ¿Cómo puedes determinar la distancia del observador 2 a la Torre a partir de la distancia del observador 1 a la misma? Expresa los cálculos necesarios.

5. ¿Cuál es la posición del observador 1 para que la altura aproximada de la torre sea la que nos interesa calcular, 5,06 m? Expresa los cálculos necesarios 
Ahora, vamos a cambiar los datos del problema. Para cada problema, manda una captura de pantalla del applet en la que muestres una solución aproximada, lo más cercana posible al al valor real de la altura de la Torre.

Problema a) Dos observadores ven una torre desde $\alpha=60^{\circ}$ y $\beta=30^{\circ}$ respectivamente. Con el pie de la torre forman $\gamma=0^{\circ}$ y están a una distancia de $8 \mathrm{~m}$ (d). ¿Cuál es la altura de la torre en metros?

Problema b) Dos observadores ven una torre desde $\alpha=60^{\circ}$ y $\beta=30^{\circ}$ respectivamente. Con el pie de la torre forman $\gamma=180^{\circ}$ y están a una distancia de $8 \mathrm{~m}$ (d). ¿Cuál es la altura de la torre en metros?

Problema c) Dos observadores ven una torre desde $\alpha=60^{\circ}$ y $\beta=30^{\circ}$ respectivamente. Con el pie de la torre forman $\gamma=45^{\circ}$ y están a una distancia de $8 \mathrm{~m}(\mathrm{~d})$. ¿Cuál es la altura de la torre en metros? 


\section{Anexo II-Cuestionario 2}

Has resuelto perfectamente el problema planteado mediante cálculos, pero nos interesa que nos muestres la resolución del problema a partir del uso del applet que se te facilita. El enlace para acceder al Applet es el siguiente: https://www.geogebra.org/m/bwretzcp

En primer lugar, te damos una serie de recomendaciones para su uso:

a) Una vez que has introducido los datos del problema en los huecos facilitados, fíjate que el observador 1 puedes moverlo libremente a lo largo del eje X y variar su distancia a la Torre ubicada en el punto A. Observa que la altura de la torre también varía, es decir, estás tomando una medida aproximada de la torre que es diferente a la real que ya la has calculado, porque el observador 1 no está en la posición adecuada. Explica cómo calcular a mano la altura de la torre aproximada en función de la distancia del observador 1 a la misma.

b) Observa que cuando mueves el observador 1 , el observador 2 va variando su distancia al punto A. Explica cómo calcular a mano la distancia del observador 2 a la torre en función de la distancia del observador 1 a la misma.

c) Fíjate por último en la circunferencia de centro el observador 1 ¿De qué radio es esa circunferencia?; ¿Dónde se sitúa el observador 2 cuando la altura calculada es exactamente la real obtenida en la resolución a mano del problema?

Por ello, os propongo que completéis las tablas siguientes y que calculéis la distancia del observador 2 a la torre y la altura de la torre a partir de diferentes distancias del observador 1 a la misma para el caso inicial, es decir, el caso en el que dos observadores ven una torre desde $\alpha=70^{\circ}$ y $\beta=30^{\circ}$ respectivamente. Con el pie de la torre forman $\gamma=60^{\circ} \mathrm{y}$ están a una distancia de $8 \mathrm{~m}(\mathrm{~d})$.

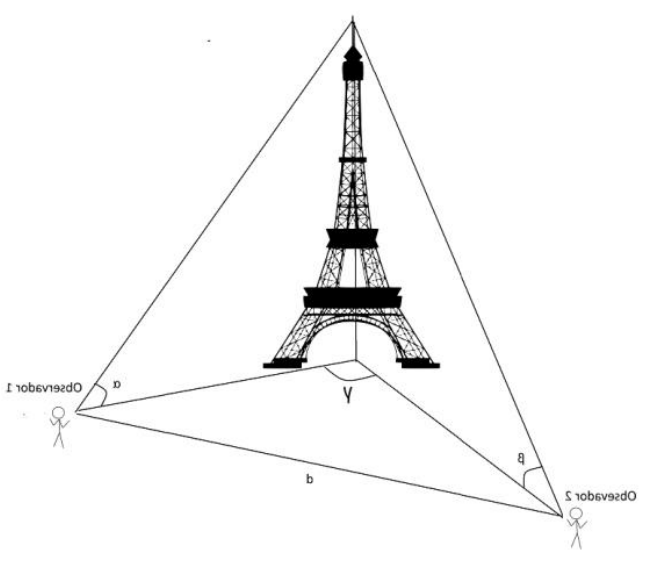




\begin{tabular}{|l|l|}
\hline $\mathrm{d}_{1}$ (Distancia del observador 1 a la Torre) & $\mathrm{d}_{2}$ (Distancia del observador 2 a la Torre) \\
\hline $2 \mathrm{~m}$ & \\
\hline $4 \mathrm{~m}$ & \\
\hline $8 \mathrm{~m}$ & \\
\hline
\end{tabular}

\begin{tabular}{|l|l|}
\hline $\mathrm{d}_{1}$ (Distancia del observador 1 a la Torre) & $\begin{array}{l}\mathrm{h} \text { (Altura de la torre vista por el } \\
\text { observador 1 con ángulo fijo } \alpha)\end{array}$ \\
\hline $2 \mathrm{~m}$ & \\
\hline $4 \mathrm{~m}$ & \\
\hline $8 \mathrm{~m}$ & \\
\hline
\end{tabular}

Ahora con todo lo nuevo que sabéis intentad resolver de nuevo los casos siguientes con la Applet y adjuntar las capturas de pantalla en las que se muestre una solución aproximada, lo más cercana posible al valor real de la altura de la Torre que ya te muestra el Applet (Altura teórica de la torre). Explicad el procedimiento que habéis utilizado. Podéis usar y completar las tablas como las anteriores si os resultan de utilidad. También podéis hacer zoom para ver valores más precisos.

Problema a) Dos observadores ven una torre desde $\alpha=60^{\circ}$ y $\beta=30^{\circ}$ respectivamente. Con el pie de la torre forman $\gamma=0^{\circ}$ y están a una distancia de $8 \mathrm{~m}(\mathrm{~d})$. ¿Cuál es la altura de la torre en metros?

Problema b) Dos observadores ven una torre desde $\alpha=60^{\circ}$ y $\beta=30^{\circ}$ respectivamente. Con el pie de la torre forman $\gamma=180^{\circ}$ y están a una distancia de $8 \mathrm{~m}(\mathrm{~d})$. ¿Cuál es la altura de la torre en metros?

Problema c) Dos observadores ven una torre desde $\alpha=60^{\circ}$ y $\beta=30^{\circ}$ respectivamente. Con el pie de la torre forman $\gamma=45^{\circ}$ y están a una distancia de $8 \mathrm{~m}(\mathrm{~d})$. ¿Cuál es la altura de la torre en metros? 